An ODE-based mixed modelling approach for B- and T-cell dynamics induced by Varicella-Zoster Virus vaccines in adults shows higher T-cell proliferation with Shingrix than with Varilrix

Peer-reviewed author version

Keersmaekers, Nina; OGUNJIMI, Benson; Van Damme, Pierre; Beutels, Philippe \& HENS, Niel (2019) An ODE-based mixed modelling approach for B- and T-cell dynamics induced by Varicella-Zoster Virus vaccines in adults shows higher T-cell proliferation with Shingrix than with Varilrix. In: VACCINE, 37(19), p. 2537-2553.

DOI: 10.1016/j.vaccine.2019.03.075

Handle: http://hdl.handle.net/1942/30031 


\title{
An ODE-based mixed modelling approach for B- and T-cell dynamics induced by Varicella-Zoster Virus vaccines in adults shows higher T-cell proliferation with Shingrix than with Varilrix
}

\author{
Nina Keersmaekers ${ }^{1,2, *}$, Benson Ogunjimi ${ }^{1,2,3,4}$, Pierre Van Damme ${ }^{2,5}$, \\ Philippe Beutels ${ }^{1,2}$, Niel Hens ${ }^{1,2,6}$
}

\section{Abstract}

Clinical trials covering the immunogenicity of a vaccine aim to study the longitudinal dynamics of certain immune cells after vaccination. The corresponding immunogenicity datasets are mainly analyzed by the use of statistical (mixed effects) models. This paper proposes the use of mathematical ordinary differential equation (ODE) models, combined with a mixed effects approach. ODE models are capable of translating underlying immunological post vaccination processes into mathematical formulas thereby enabling a

\footnotetext{
${ }^{*}$ Corresponding author

Email address: nina.keersmaekers@uantwerp.be (Niel Hens)

${ }^{1}$ Centre for Health Economics Research \& Modeling Infectious Diseases (CHERMID), Vaccine \& Infectious Disease Institute (VAXINFECTIO), University of Antwerp, Antwerp, Belgium

${ }^{2}$ Antwerp Unit for Data Analysis and Computation in Immunology and Sequencing (AUDACIS), University of Antwerp, Antwerp, Belgium

${ }^{3}$ Antwerp Center for Translational Immunology and Virology (ACTIV), Vaccine \& Infectious Disease Institute (VAXINFECTIO), University of Antwerp, Antwerp, Belgium

${ }^{4}$ Department of Paediatrics, Antwerp University Hospital, Edegem, Belgium

${ }^{5}$ Centre for the Evaluation of Vaccination (CEV), Vaccine \& Infectious Disease Institute (VAXINFECTIO), University of Antwerp, Antwerp, Belgium

${ }^{6}$ Interuniversity Institute for Biostatistics and statistical Bioinformatics, Hasselt University, Diepenbeek, Belgium
} 
testable data analysis. Mixed models include both population-averaged parameters (fixed effects) and individual-specific parameters (random effects) for dealing with inter- and intra-individual variability, respectively.

This paper models B-cell and T-cell datasets of a phase I/II, open-label, randomized, parallel-group study (NCT00492648) in which the immunogenicity of a new Herpes Zoster vaccine (Shingrix) is compared with the original Varicella Zoster Virus vaccine (Varilrix).

Since few significant correlations were found between the B-cell and T-cell datasets, each dataset was modeled separately. By following a general approach to both the formulation of several different models and the procedure of selecting the most suitable model, we were able to propose a mathematical ODE mixed-effects model for each dataset. As such, the use of ODE-based mixed effects models offers a suitable framework for handling longitudinal vaccine immunogenicity data. Moreover, this approach allows testing for differences in immunological processes between vaccines or schedules. We found that the Shingrix vaccination schedule led to a more pronounced proliferation of T-cells, without a difference in T-cell decay rate compared to the Varilrix vaccination schedule.

Keywords: Mathematical, models, ordinary differential equations, ODE, mixed effects, Varicella Zoster Virus, VZV, Herpes Zoster, vaccines, B-cells, T-cells, dynamics

\section{1. Introduction}

2 Vaccines are developed in order to activate (and subsequently cause pro3 liferation) of B-cells and T-cells that are specifically directed against the 
4 vaccine antigens. B-cells will (1) produce antigen-specific antibodies and (2) 5 differentiate into long-living plasma cells. Antibodies are the primary ef6 fectors of the so-called humoral immune response in combating circulating 7 pathogens. T-cells represent the cellular immune response and consist of 3 CD4+ T-cells and CD8+ T-cells (and some other classes not discussed in 9 this paper). CD4+ T-cells have an important role in helping other cell types (such as B-cells and macrophages) combating pathogens. CD8+ T-cells have a direct cytotoxic function and can target host cells that are infected by a pathogen.

The vaccine-induced B-cells and T-cells are hypothesized to be capable of pre-

venting or minimizing the morbidity related to the infectious disease against which the vaccine is targeted. Vaccine immunogenicity trials aim to study the longitudinal dynamics of the specific immune response following vaccination. These trials can range from several months to several decades. The quantitative analysis of longitudinal immune response data has evolved from between-group and time point comparisons to statistical regression analyses [1, 2, 3]. Current state-of-the art statistical analyses of longitudinal data consist of a mixed effects model approach in which a separation is made between population-averaged parameters (so called fixed effects) and individual-specific parameters (so called random effects). More recently, Andraud et al. [4] and Le et al. [5] published the first papers in which the mixed effects modeling approach was combined with the use of ordinary differential equations (ODE), thereby more closely resembling immune response dynamics post vaccination. Whereas [4] focused on the long term dynamics following vaccination, [5] focused on the short term dynamics following 
vaccination.

ODE-based mathematical models are capable of translating the underlying immunological/biological theory into a testable data analysis. Moreover, the combination with mixed effects modeling offers a methodology capable of dealing with inter- and intra-individual variability. As such, the use of ODE-based mixed effects models offers a suitable framework for handling longitudinal vaccine immunogenicity data.

In this paper, we set out to use ODE-based mixed effects models to study B-cell and T-cell dynamics following varicella-zoster virus (VZV) vaccinations in VZV-immune adults. In particular, this framework will allow us to disentangle the immunogenic differences between two different VZV-specific vaccines.

We start by showing the immunogenicity data from two VZV vaccine studies consisting of B-cells and CD4+ T-cells of participants at different time points. We then present the differential equations, the ODE and the ODEbased mixed effects models used to describe the immune response dynamics within each individual as well as the associated model selection procedures. By applying the above methods, we consequently select a suitable model for each dataset. Next, we compare the results of the two VZV vaccines, using a group-related effect on a chosen parameter. Correlations in and between the datasets are also explored. We end by reviewing our findings, discussing shortcomings and adding points for future research. 


\section{Materials and methods}

\subsection{Data}

The phase I/II, open-label, randomized, parallel-group study EXPLOCR004 (101501) investigated the safety and immunogenicity of an adjuvanted recombinant glycoprotein E vaccine ("HZ/su", GSK) for VZV, by comparing it with a live attenuated Oka strain VZV vaccine ("OKA", Varilrix ${ }^{\circledR}$, GSK). To evaluate safety prior to administration in older adults, two groups of young adults (18-30 years) were vaccinated with two vaccine doses two months apart. The first group (GROUP 1; sample size: $n_{1}=10$ ) received one dose of $\mathrm{HZ} / \mathrm{su}$ and one dose of OKA concomitantly at month 0 and month 2 (i.e. four doses in total), whereas the second group (GROUP 2; $\left.n_{2}=10\right)$ received a dose of $\mathrm{HZ} / \mathrm{su}$ both times (i.e. two doses in total). After vaccine safety was confirmed, three groups of older adults (50-70 years) were vaccinated two months apart, one group (GROUP $3 ; n_{3}=45$ ) received twice a single dose of $\mathrm{HZ} / \mathrm{su}$, the second (GROUP $4 ; n_{4}=45$ ) twice a single dose of OKA and the last (GROUP $5 ; n_{5}=45$ ) twice two concomitant doses of HZ/su and OKA. So, all in all, 155 participants were divided over these 5 groups. The properties of each group are summarized in Table 1.

Safety and immunogenicity were assessed for all groups up to 12 months post-vaccination in the original study. In order to obtain long-term immunogenicity data on the newly proposed $\mathrm{HZ} / \mathrm{su}$ vaccine, 23 individuals from the groups solely receiving HZ/su (i.e. GROUPS 2 and 3) were assessed up to 42 months post-vaccination in the extension studies NCT00492648. Every individual was considered a responder. Descriptive statistical tests were used to assess the difference between the two vaccines. A significant higher immune 


\begin{tabular}{|l||l|l|l|l|}
\hline GROUP & $\begin{array}{l}\text { sample } \\
\text { size }\end{array}$ & age & vaccine & schedule \\
\hline \hline 1 & 10 & $18-30$ & $\mathrm{HZ} / \mathrm{su}+\mathrm{OKA}$ & $1+1$ dose mo. $0,1+1$ dose mo. 2 \\
\hline 2 & 10 & $18-30$ & $\mathrm{HZ} / \mathrm{su}$ & 1 dose mo. 0,1 dose mo. 2 \\
\hline 3 & 45 & $50-70$ & $\mathrm{HZ} / \mathrm{su}$ & 1 dose mo. 0,1 dose mo. 2 \\
\hline 4 & 45 & $50-70$ & $\mathrm{OKA}$ & 1 dose mo. 0,1 dose mo. 2 \\
\hline 5 & 45 & $50-70$ & $\mathrm{HZ} / \mathrm{su}+$ OKA & $1+1$ dose mo. 0, $1+1$ dose mo. 2 \\
\hline
\end{tabular}
sample size, age, vaccine and vaccination schedule (mo.=month). Group 4 is defined as reference group. the group solely receiving the OKA vaccine. We refer to [6 for a more in depth description of the design and results of these studies

\section{Table 1: Properties of the different groups in the VZV vaccine trial. Shown are}

\subsubsection{B-cell data}

First, we used data on the number of antigen-specific memory B-cells, provided by a B-cell ELISPOT assay, at baseline, and at 1 month and 12 months after receiving the first vaccine dose. Two tests were performed: the first used Varilrix $\left.{ }^{(}\right)(1 / 20 \mathrm{x})$ as stimulus in the B-cell ELISPOT assay, the second used $100 \mu \mathrm{l}$ of $\mathrm{gE}(10 \mu \mathrm{g} / \mathrm{ml})$ as stimulus. This resulted in two datasets comprising the frequencies of either "total" VZV-specific memory Bcells or gE-specific memory B-cells per million of total memory B-cells. The participants were split into 5 different groups, based on age and vaccine type (see Section 2.1), and their profiles relative to the total number of memory B-cells are plotted in Fig. 1 for the Varilrix-specific B-cells.

response was found in the groups receiving the $\mathrm{HZ} / \mathrm{su}$ vaccine compared to 

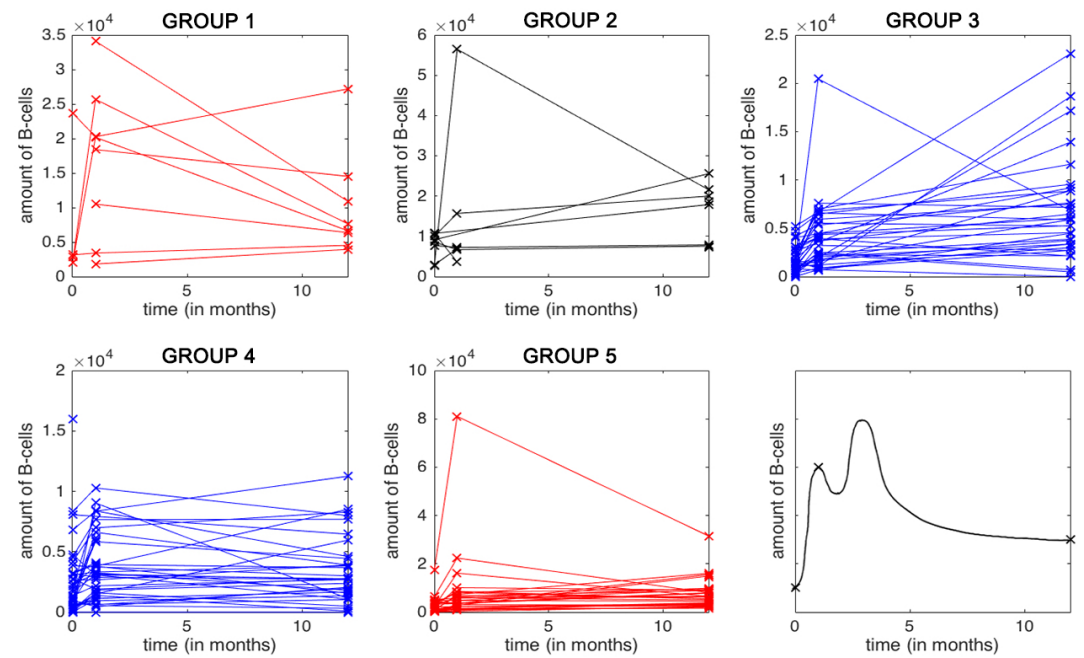

Figure 1: Amount of VZV-specific memory B-cells. Measured by B-cell ELISPOT (Varilrix stimulus) per $10^{6}$ total memory cells, up to 12 months.

Data are shown per study group. The last panel acts as an illustration of the vaccination dynamics and shows a hypothetical, smooth function of the expected change in number of memory B-cells over time (in months), based on the observed data points per individual and considering the second vaccination at month 2 . 
We observe an increase in memory B-cells (further denoted as B-cells), after vaccination at time $t=0$ months. At time $t=2$ months, the subjects were re-vaccinated, but no data were collected at that time point. Fig 1 shows only the time points for which data were available $(t=0,1$ and 12 months). Since it is reasonable to expect a (higher) peak in the data after the second dose at $t=2$ months, we will assume a time period $[0, h], h>0$ during which the level of B-cells increases up to a point, $h$, after which it decreases. The data plots of gE-specific memory B-cells show a similar pattern (see Supplementary material, Fig S1).

\subsubsection{T-cell data}

Intracellular cytokine staining (ICS) in combination with a flow cytometric readout was performed to measure the amount of CD4+ T-cells that produced at least 2 cytokines (interferon-gamma, interleukin 2, CD40 Ligand, tumor necrosis factor alpha) using both Varilrix and $\mathrm{gE}$ as stimuli (in separate experiments). The subsequent two datasets comprise the same 155 participants, but now with time points at baseline, and at 1, 2, 3 and 12 months after receiving the first vaccine dose. The total VZV-specific T-cell profiles of the participants by study group are shown in Fig 2 .

Given that T-cell data were collected at more time points than B-cell data, we now observe a second peak in the group-specific data plots, as is expected given the vaccine administrations at month 2 . Therefore we will use two time periods $\left[0, h_{1}\right]$ and $\left[2, h_{2}\right]$ (with $0<h_{1}<2<h_{2}$ ) during which the level of T-cells first increases and then decreases. In case only one peak is observed, we will assume $h_{1}=2<h_{2}$.

As with the B-cell profiles, the gE specific T-cell profiles are shown in Fig 

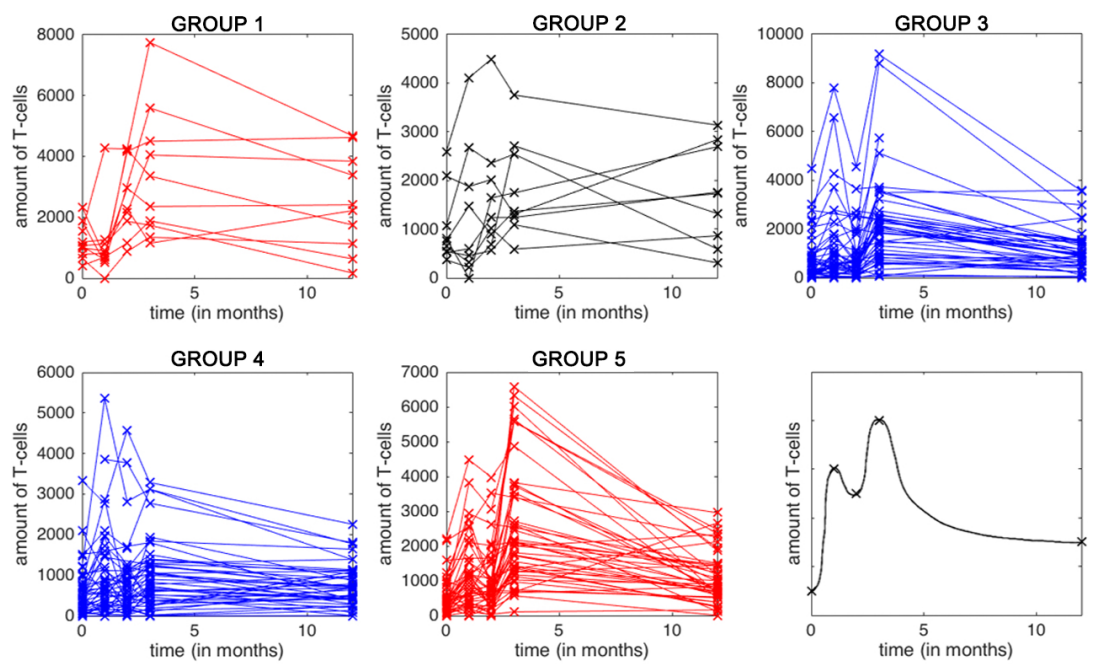

Figure 2: Amount of VZV-specific CD4+ T-cells, producing at least 2 immune markers. Measured by ICS per $10^{6} \mathrm{CD} 4+$ T-cells, shown per group and up to 12 months. The last panel acts as an illustration of the vaccination dynamics and shows a hypothetical, smooth function of the expected change in number of CD4+ T-cells over time (in months), based on the observed data points per individual. 
S2.

\subsection{Mathematical methods}

We used systems of (nonlinear) ODEs to model the B-cell and T-cell dynamics. We applied a systematic approach to fit and compare several models in order to obtain the models that best describe the available data, while providing sufficient biological interpretation. The detailed version of all ODEs, along with their solution, can be found in Appendix $A$. In the following subsections we provide the basic rationale of these ODEs for both B-cell and T-cell dynamics, respectively.

\subsubsection{B-cell dynamics models}

We describe the dynamics of the memory B-cells using the following ODE:

$$
\frac{d B}{d t}=f_{1}(B) I_{t \leq h}-f_{2}(B)
$$

where $B_{0}=B(0)$ denotes the initial number of memory B-cells at time $=0$ (months) and $f_{1}$ and $f_{2}$ are smooth functions of the number of memory Bcells at time $t$ (months), describing the change in the number of B-cells due to activation and decay of B-cells. We assume that the activation of B-cells happens during a certain time period $[0, h]$ and that after this time period, no new B-cells are activated. The process of activation of B-cells is described by the function $f_{1}$. The decay in the number of B-cells occurs at all times and is described by $f_{2}$. In all models, the decay rate is assumed to remain constant over time.

A first distinction between models can be made through the nature of the rate at which B-cells will be activated. A schematic overview of the different 
choices in activation/proliferation functions is given in Fig 3. In model B1, the proliferation rate is assumed to be constant over the time period $[0, h]$. Another option is to assume a rate which is proportional to the number of B-cells at time $t$, as in model B2.

A distinction can be made between memory B-cells with a short lifespan and B-cells with a longer lifespan. A similar line of reasoning is followed in [7], in which a distinction is made between memory B-cells and long living plasma cells. Models B3 and B4 incorporate this distinction by including different equations in the ODE system for short living B-cells (SB) and long living B-cells (LB). The dynamics of the SB in models B3 and B4 are similar to those in model B1, but at time 0 months, no SB are present in models B3 and B4. LB however, are present in models B3 and B4 at time 0 months. To distinguish between models B3 and B4, the dynamics of LB are considered. First, in view of their long lifespan, no decay of LB is assumed and model B3 expresses no proliferation rate either which means that the total number of LB remains constant over time. Second, in model B4, a constant proliferation rate of LB is introduced during time period $[0, h]$ after which their number will remain constant over time. We refer to Appendix B for an overview of the parameters used in the dynamic B-cell models.

\subsubsection{T-cell dynamic models}

The design of the T-cell models follows a similar procedure as that of the B-cell models. The following ODE describes the basic dynamics of the stimulus-specific T-cell population:

$$
\frac{d T}{d t}=f_{1}(T) I_{0 \leq t \leq h_{1}}+f_{2}(T) I_{2 \leq t \leq h_{2}}-f_{3}(T),
$$




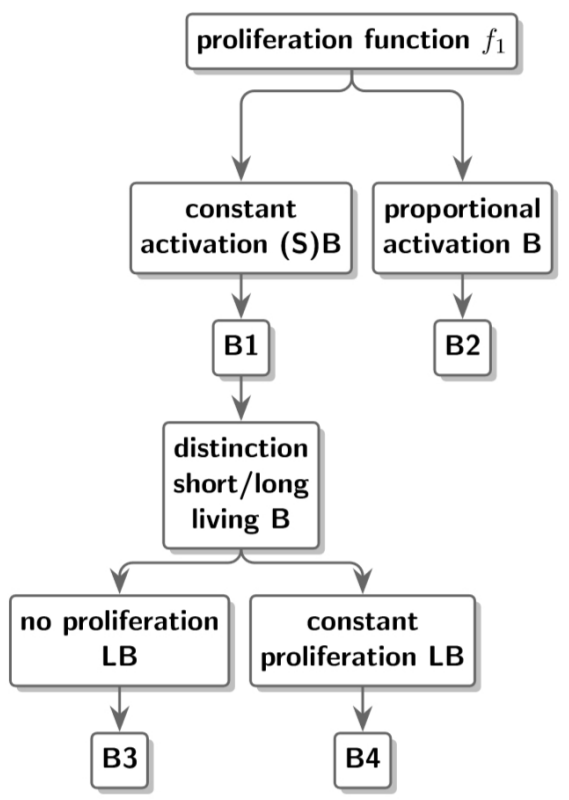

Figure 3: Schematic representation of the different proliferation functions used to describe the B-cells population. 
with $T_{0}=T(0)$ the number of T-cells at time 0 (months). In this equation, $f_{1}(T)$ describes the proliferation of T-cells after the first vaccination event at time 0 , which will occur until a certain time point $h_{1}$ (with $0<h_{1} \leq 2$ ). Afterwards, no T-cells will be activated until the second vaccination event 2 months after the first, which $f_{2}(T)$ describes as the proliferation of T-cells during the time period, $\left[2, h_{2}\right]$, with $h_{2}$ the time point at which the second peak in T-cells is reached. The decay of T-cells will happen during the whole time period, and is represented by the function $f_{3}(T)$. In all models, it will be assumed that the decay rate of T-cells remains constant over time (cf. B-cell models). Moreover, we assume that the activation of T-cells after each vaccination event happens according to a constant proliferation rate. It is noteworthy that a non-constant proliferation rate, proportional to the number of T-cells, was part of exploratory analyses, but these explorations did not result in a convergent model (see the Inference and model selection section).

The rates after the first and second vaccination event are not necessarily equal and neither are the ranges of the time periods $\left[0, h_{1}\right]$ and $\left[2, h_{2}\right]$. Since T-cells are still present in the blood at the time of the second vaccination, we assume a different number of new T-cells will be activated.

Fig 4 summarizes the difference between all T-cell models we will consider. We start by assuming that all T-cells can be regarded as one population. With the additional assumptions that $f_{1}$ and $f_{2}$ are identical functions (and that the proliferation rates of T-cells are equal after each vaccination), we arrive at model T1. Model T2 does not presume both proliferation rates are equal. 


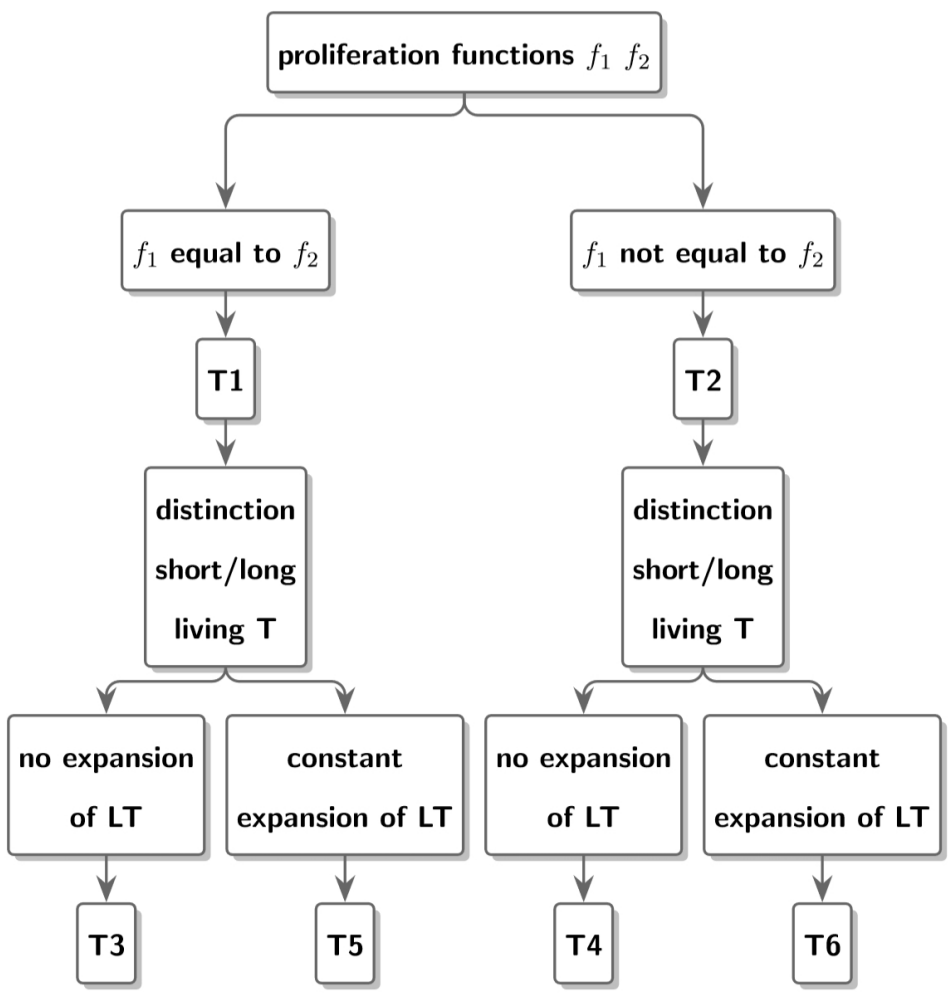

Figure 4: Schematic representation of the difference in proliferation functions used to describe the T-cell dynamics. 
Next, models are considered in which the T-cell population is divided into short living and long living T-cell populations, represented by ST and LT, respectively. This can be considered as a distinction between effector T-cells (short living) and memory T-cells (long living).

The dynamics of the short living T-cells are similarly described as in models T1 and T2: a constant number of short living T-cells will be activated after each vaccination (not necessarily the same number), while the decay of short living T-cells occurs at all times at a constant decay rate. For models T3 and T4, the assumption is made that the total number of long living Tcells remains constant over time. If we add the distinction between models with equal and different functions $f_{1}$ and $f_{2}$, we arrive at models T3 and T4, respectively. Adding proliferation rates of long living T-cells after each vaccination, models T3 and T4 are extended to yield models T5 and T6. In order to restrict the total number of parameters, the long living $\mathrm{T}$ cell proliferation rates are assumed to be equal. We refer to Appendix B for an overview of the parameters used in the dynamic T-cell models.

\subsection{Nonlinear mixed models}

The dynamic models described in previous subsections can be formulated as nonlinear mixed models in which the parameters are assigned distributions through the specification of fixed and random effects. The fixed component can be interpreted as a population parameter, i.e. an average for all individuals, while the random component accounts for individual differences. More specifically, each individual parameter $P_{i}$ can thus be written as $P_{i}=u_{i} \times P_{\text {pop }}$ where $P_{\text {pop }}$ is a population parameter and $u_{i}$ is log-normally distributed with $\mathrm{E}\left(u_{i}\right)=1$. 
In case of the presence of a categorical variable (e.g. different group in vaccine trial study), the different groups can be compared against each other by adding a component $\beta_{j}$ to the distribution of a certain parameter. $\beta_{j}$ describes how for group $j$, this parameter deviates from the (chosen) reference group. This makes it possible to test whether one group has a significant higher variable (e.g. rate of cell activation) compared to the reference group. The Monolix software ${ }^{(C)}$ Lixoft was used for the estimation of the parameters. A built in stochastic approximation of the standard expectation maximization algorithm (SAEM) with simulated annealing, combined with a Markov Chain Monte Carlo (MCMC) procedure which replaces the simulation step of the SAEM algorithm, is used to obtain population parameters estimates. Loglikelihood calculation was done by importance sampling, in which a fixed $t$-distribution is assumed with 5 degrees of freedom. For more details on the algorithms used we refer to [8]. Mostly, Monolix default parameter values were used in the algorithms (see Appendix C) The two-step SAEM-MCMC algorithm uses $10^{6}+10^{5}$ iterations in order to assess convergence for estimating the population parameters.

\subsection{Inference and model selection}

Although mathematical identifiability is guaranteed for the models presented in the Mathematical methods section, the complexity of these models when combining them with many random effects in view of the data limitations in terms of sampling times and sample sizes resulted in non-convergence. Therefore, simplifying assumptions needed to be made. One such simplifying assumption is presuming that the decay of $\mathrm{B}$ or T-cells is identical for all individuals, implying that the random effect for that decay parameter is 
omitted from the model.

For both the B-cell and T-cell data sets, the following procedure was used for comparing and selecting the most suitable biologically plausible model to describe the data. In a first step, a list of models was composed, consisting of models B1 to B4 for the B-cell data and of models T1 to T6 for T-cell data, together with assumptions on the parameters reflecting whether or not individual variation on these parameters is present, i.e. whether or not random effects were included for the different parameters. The model parameters were then estimated with the Monolix software.

Models with poor SAEM convergence, likely because of abundant model complexity, were discarded. Next, the candidate models were compared using Akaike's Information Criterion (AIC) and the model with lowest AIC value was selected as first candidate model. Subsequently, a non-parametric bootstrap, using 1000 bootstrap re-samples, was performed on the candidate model. Since a sequential approach based on the candidate models with the lowest AIC values was used, the need to perform bootstraps for all candidate models was avoided, in order to decrease the number of computations. It was found that for a bootstrap, either $65 \%-77 \%$ of the samples had proper SAEM convergence, or the proportion of bootstrap samples with proper convergence was less than $15 \%$. For this reason the criterion for good bootstrap convergence was defined as having at least $65 \%$ of bootstrap samples with proper SAEM convergence. In case of poor bootstrap convergence, the candidate model was rejected from the list of candidate models.

Next, a sensitivity analysis on the bootstrap results of the (converging) candidate model was performed by investigating whether the presence or absence 
of certain profiles of individuals in the bootstrap samples, had influence on its model convergence. For instance, if a single participant's profile was more frequently part of non-converging datasets, a new bootstrap was performed, excluding the specific participant's profile. Again, in case of poor bootstrap convergence the candidate model was rejected. If convergence remained sufficiently robust, it was investigated whether it was possible to improve the biological plausibility of the candidate model. This was done by examining the assumptions made on the parameters and adjusting those. An example of this is shown in the Model selection of T-cell datasets results section. The new model then became a candidate model.

\subsection{B-cell and T-cell dynamics associations}

As CD4+ T-cells may directly influence the activation of B-cells, we investigated the existence of associations between B-cell and T-cell dynamics. We used a raw data complete cases analysis given that constructing a joint model based on the same concepts was not successful, likely due to data limitations (Inference and model selection section).

More specifics on this analysis, and its results, can be found in Appendix D.

\section{Results}

\subsection{Model selection of B-cell datasets}

We started by modeling the Varilrix-specific B-cell dataset, for which the model selection procedure outlined in the Inference and model selection section was followed. All details on the considered models and the following of the procedure can be found in Appendix E. The resulting Varilrix-specific 
(1)

B-cell model differentiates between $\mathrm{SB}$ and LB, LB are assumed to remain constant through time (model B3, see B-cell dynamics models section. In time period $[0, h]$, a constant number of SB is activated and this proliferation rate is considered a group-specific parameter. The decay rate is assumed to be equal for each individual.

The estimated population parameters of the selected B-cell model are shown in Table 2.

\begin{tabular}{|l|l|l|}
\hline parameter & estimate & $\mathbf{9 5 \%}$ CI \\
\hline \hline$L B_{0}$ & 1852.84 & $(1425.79,2407.80)$ \\
\hline$a S B$ & 774.17 & $(367.67,1630.12)$ \\
\hline$\beta_{1}$ & 2.14 & $(0.79,3.49)$ \\
\hline$\beta_{2}$ & 1.44 & $(-0.02,2.91)$ \\
\hline$\beta_{3}$ & 0.67 & $(-0.12,1.46)$ \\
\hline$\beta_{5}$ & 0.90 & $(0.02,1.79)$ \\
\hline$u S B$ & 0.13 & $(0.11,0.16)$ \\
\hline$h$ & 3.30 & $(2.05,5.30)$ \\
\hline
\end{tabular}

Table 2: Varilrix-specific B-cell results. Parameter estimates and corresponding $95 \%$ confidence intervals (CI) of final model B3b. $S B(0)$ is assumed to be zero, $L B_{0}=L B(0)$ denotes the initial number of LB. The proliferation of SB is constant in time period $[0, h]$, at rate $a S B$ and assumed to be group-specific. $\beta_{i}(i=1,2,3,5)$ represents the increase of $a S B$, compared to the reference group $4\left(\beta_{4}=0\right)$. Decay of SB happens at rate $u S B$. The number of LB remains constant through time.

The same models as with the Varilrix-specific B-cell data were used for the gE-specific B-cell data and a similar model selection procedure was followed. The outcome of the model selection was a model which does not differentiate 
between SB and LB. In time period $[0, h]$, a constant number of B-cells are activated (model B1). All parameters are assumed to have random effects and the activation rate of B-cells is chosen as group-specific parameter. The parameter estimations, along with confidence interval, are shown in Table 3.

\begin{tabular}{|l|l|l|}
\hline parameter & estimate & $\mathbf{9 5 \%}$ CI \\
\hline \hline$B_{0}$ & 564.39 & $(429.80,741.14)$ \\
\hline$a B$ & 566.08 & $(337.69,948.95)$ \\
\hline$\beta_{1}$ & 2.59 & $(1.52,3.66)$ \\
\hline$\beta_{2}$ & 2.13 & $(1.08,3.17)$ \\
\hline$\beta_{3}$ & 1.27 & $(0.69,1.84)$ \\
\hline$\beta_{5}$ & 1.25 & $(0.59,1.91)$ \\
\hline$u B$ & 0.035 & $(0.0060,0.21)$ \\
\hline$h$ & 2.51 & $(1.78,3.52)$ \\
\hline
\end{tabular}

Table 3: gE-specific B-cell results. Parameter estimates and corresponding 95\% confidence intervals (CI) of final model B1a. No distinction between SB and LB is presumed. $B_{0}=B(0)$ denotes the initial number of B-cells. The proliferation of B-cells is constant in time period $[0, h]$, at rate $a B$ and assumed to be group-specific. $\beta_{i}(i=1,2,3,5)$ represents the increase of $a B$, compared to the reference group $4\left(\beta_{4}=0\right)$. Decay of B-cells happens at rate $u B$.

\subsection{Model selection of T-cell datasets}

a By following the T-cell model selection procedure (details in Appendix E), a model which differentiates between ST and LT, was selected as final Varilrix-specific T-cell model. This model furthermore assumes the number of LT remains constant through time and assumes activation of ST is constant in time periods $\left[0, h_{1}\right]$ and $\left[2, h_{2}\right]$ with $a_{2} S T=0.15 \times a_{1} S T$ (model T3, see 
T-cell dynamics models section). Moreover, all parameters are assumed to have random effects with the activation rate a group-specific parameter. The model parameter estimates are shown in Table 4.

\begin{tabular}{|l|l|l|}
\hline parameter & estimate & 95\% CI \\
\hline \hline$L T_{0}$ & 517.53 & $(422.96,633.25)$ \\
\hline$a S T$ & 1825.20 & $(893.64,3727.84)$ \\
\hline$\beta_{1}$ & 1.50 & $(0.29,2.72)$ \\
\hline$\beta_{2}$ & 0.76 & $(-0.81,2.32)$ \\
\hline$\beta_{3}$ & 1.57 & $(0.90,2.25)$ \\
\hline$\beta_{5}$ & 1.73 & $(1.07,2.39)$ \\
\hline$u S T$ & 0.40 & $(0.18,0.86)$ \\
\hline$h_{1}$ & 0.026 & $(0.0088,0.075)$ \\
\hline$h_{2}$ & 3.85 & $(2.57,5.78)$ \\
\hline
\end{tabular}

Table 4: Varilrix-specific T-cell results. Parameter estimates and corresponding $95 \%$ confidence intervals (CI) of final model T3a'. $S T(0)$ is assumed to be zero, $L T_{0}=L T(0)$ denotes the initial number of LT. The proliferation of ST is constant in time period $\left[0, h_{1}\right]$ at rate $a S T$ and in time period $\left[2, h_{2}\right]$ at rate 0.15.aST. aST is assumed to be groupspecific with effects $\beta_{i}(i=1,2,3,5)$, representing the increase of $a S T$, compared to the reference group $4\left(\beta_{4}=0\right)$. Decay of ST happens at rate $u S T$. The number of LT remains constant through time.

The same T-cell models were used in the model selection procedure of the gE-specific T-cell data. The parameter estimations of the final $\mathrm{gE}$-specific T-cell model are shown in Table 5. This model does not differentiate between ST and LT and assumes a constant activation of T-cells in time periods $\left[0, h_{1}\right]$ and $\left[2, h_{2}\right]$ with $a_{2} S T=0.66 \times a_{1} S T$ (model T1). All parameters were assumed to have random effects with the activation rate being a group-specific 
parameter. Moreover, individuals 89 and 149 were viewed as statistical outliers (with datapoints distant from other individuals) and their profiles were left out of this dataset.

\begin{tabular}{|l|l|l|}
\hline parameter & estimate & $\mathbf{9 5 \%}$ CI \\
\hline \hline$T_{0}$ & 90.07 & $(45.31,179.07)$ \\
\hline$a T$ & 329.14 & $(162.70,665.81)$ \\
\hline$\beta_{1}$ & 2.41 & $(1.75,3.32)$ \\
\hline$\beta_{2}$ & 2.30 & $(1.65,3.21)$ \\
\hline$\beta_{3}$ & 2.53 & $(1.93,3.31)$ \\
\hline$\beta_{5}$ & 2.43 & $(1.83,3.22)$ \\
\hline$u T$ & 0.54 & $(0.26,1.11)$ \\
\hline$h_{1}$ & 0.21 & $(0.15,0.31)$ \\
\hline$h_{2}$ & 9.03 & $(5.61,14.53)$ \\
\hline
\end{tabular}

Table 5: gE-specific T-cell results. Parameter estimates and corresponding 95\% confidence intervals (CI) of final model T1a'. No distinction of ST and LT is presumed. $T_{0}=T(0)$ denotes the initial number of T-cells. The proliferation of T-cells is constant in time period $\left[0, h_{1}\right]$ at rate $a T$ and in time period $\left[2, h_{2}\right]$ at rate $0.66 \times a T$. $a T$ is assumed to be group-specific with effects $\beta_{i}(i=1,2,3,5)$, representing the increase of $a T$, compared to the reference group $4\left(\beta_{4}=0\right)$. Decay of T-cells happens at rate $u T$.

\subsection{Vaccine differences}

Group-specific effects on chosen parameters make it possible to compare each group by examining the differences in these effects. This comparison focuses on different proliferations of B- and T-cells. Group-specific effects were also added on other parameters, more specifically the decay rate and time point $h$, which marks the end of the proliferation period after vaccina- 
tion. However, models with these group-specific parameters did either not have an increased AIC compared to a model without this effect, or did not show SAEM convergence.

Since a group-specific component was added to the activation of B-/T-cells for each final model, it was subsequently possible to examine whether the $\mathrm{HZ} / \mathrm{su}$ vaccine caused a higher increase in B- and or T-cells after vaccination, compared to the original OKA vaccine.

As a reminder, Table 1 summarizes the characteristics of the 5 different groups in the vaccine trial. Group 4 received the original OKA vaccine and was thereby defined as reference group.

Next, we calculated corresponding p-values of the group-specific parameters that were estimated in the Model selection of B-cell datasets and T-cell datasets sections, shown in Table 6. In view of the sample size of groups 1 and 2 (and age), we were mainly interested in $\beta_{3}$ and $\beta_{5}$. A $\beta_{i}$ higher than zero indicates a higher activation rate of cells in the groups receiving the $\mathrm{HZ} /$ su vaccine, compared to the activation rate in the reference group which received the OKA vaccine. In the case of Varilrix- and gE-specific T-cells, both groups 3 and 5 showed a significant higher activation rate $(p<0.05)$. The activation rate of gE-specific B-cells also was significantly higher compared to the reference group. Varilrix-specific B-cells also seemed to have a higher proliferation rate, though in the case of groups receiving solely the $\mathrm{HZ} / \mathrm{su}$ vaccine, not significantly so $(p>0.05)$.

As age has an important influence on vaccine responses, it was considered to compare the young groups (groups 1 and 2) to the older groups with the same vaccination schedule (groups 3 and 5). However, we can observe from 


\begin{tabular}{|c||c|c|c|c|}
\hline & Varilrix B-cells & gE B-cells & Varilrix T-cells & gE T-cells \\
\hline \hline$\beta_{1}(\mathrm{CI})$, & $2.14(0.79,3.49)$ & $2.59(1.52,3.66)$ & $1.50(0.29,2.72)$ & $2.41(1.75,3.32)$ \\
p-value & $2.8 \times 10^{-4}$ & $<10^{-5}$ & $3.8 \times 10^{-4}$ & $<10^{-5}$ \\
\hline$\beta_{2}(\mathrm{CI})$, & $1.44(-0.02,2.91)$ & $2.13(1.08,3.17)$ & $0.76(-0.81,2.32)$ & $2.30(1.65,3.21)$ \\
p-value & 0.013 & $<10^{-5}$ & 0.12 & $<10^{-5}$ \\
\hline$\beta_{3}(\mathrm{CI})$ & $0.67(-0.12,1.46)$ & $1.27(0.69,1.84)$ & $1.57(0.90,2.25)$ & $2.53(1.93,3.31)$ \\
p-value & 0.10 & $10^{-5}$ & $<10^{-5}$ & $<10^{-5}$ \\
\hline$\beta_{5}(\mathrm{CI})$ & $0.90(0.02,1.79)$ & $1.25(0.59,1.91)$ & $1.73(1.07,2.39)$ & $2.43(1.83,3.22)$ \\
p-value & 0.025 & $3 \times 10^{-5}$ & $<10^{-5}$ & $<10^{-5}$ \\
\hline
\end{tabular}

Table 6: Differences in proliferation rates per group. Group-specific parameter estimates, corresponding 95\% confidence intervals (CI) and p-values, calculated for Varilrix

B-cell, gE B-cell, Varilrix T-cell and gE T-cell data. eters 1-5 overlap. Therefore, no conclusions can be made in that respect. It is to be noted that the young cohorts are much smaller than the old cohorts ( $\mathrm{n}=10$ vs. $\mathrm{n}=45$ respectively).

\section{Discussion}

In this study we used a nonlinear mixed modeling approach using ordinary differential equations (ODE) to describe B-cell and T-cell dynamics in adults following a 2-dose vaccination against VZV by means of the novel subunit VZV gE vaccine (Shingrix, GSK) and the live-attenuated VZV vaccine (Varilrix, GSK).

Whereas the latter vaccine was not intended to be used in a similar manner as Shingrix to augment protection against herpes zoster (HZ), we did use

Tables 2, 3, 4 and 5 that the confidence intervals of the group-specific param- 
the data available from the clinical trial performed by Leroux-Roels et al (Leroux-Roels2012). In this trial, Shingrix and Varilrix were compared in regard to safety and immunogenicity in adults. Using comparative groupwise statistical tests, they found that gE-specific CD4+ T-cell levels were much higher in the groups receiving Shingrix than those receiving Varilrix alone from 3 until 42 months after vaccination. Additionally, they showed that the addition of Varilrix to Shingrix did not significantly increase the immunogenicity.

Our study was motivated by the difficulties in attributing differences between vaccines and vaccination schedules to underlying immunological processes when using the "classical" group-wise comparative statistical techniques that do not take into account underlying immunological processes.

Recently, 4] and [5] showed that nonlinear ODE mixed modeling was able to produce (plausible) estimates on several biological parameters in the setting of vaccinations.

In our study, we assessed Varilrix and VZV gE-specific B-cell and T-cell responses elicited by the 2-dose vaccination schedule for three different schedules (Shingrix only, Varilrix only and the combination of Shingrix and Varilrix on both vaccination moments). We developed and used a suitable methodological framework to obtain for each setting (immune response and vaccination schedule) the most optimal ODE model, informed by immunological theory while acknowledging data sparseness by adjusting the inferential method using a nonparametric bootstrap approach. Using this robust approach we were able to conclude that the best models did not have an overly complex structure. Models with constant proliferation rates (after each vaccination, 
in time periods $[0, h]$ (B-cells) and $\left[0, h_{1}\right]$ and $\left[2, h_{2}\right]$ (T-cells)) had lower AIC compared to models with proportional proliferation rates. Restricting the number of parameters, either by not making a distinction between short and long living B-/T-cells, or by assuming the number of long living B-/T-cells remains constant through time, resulted in models that were preferable compared to the other considered model structures. Although some of these other models may have had a more intuitively logical biological interpretation, they often did not have SAEM or bootstrap convergence.

Importantly, this way of modeling allowed us to directly compare specific parameters between several vaccination schedules. We found that the Shingrix vaccination schedules led to a more pronounced proliferation of T-cells, however without a difference in T-cell decay rate between Shingrix and Varilrix vaccination schedules. This novel result underscores the benefit of using mathematical mixed models that are based on the underlying immunological processes instead of performing standard group-wise comparisons. Indeed, in the latter case it is possible to prove significant differences between vaccines, however, it is impossible to determine what drives these differences. That is, the standard group-wise comparisons cannot show whether higher response levels are the result of either a higher proliferation of cells, a lower decay (mainly in the case of a restricted number of data points), or a longer time period $[0, h]$ in which cells are activated.

We note that the adjuvant used for the Shingrix vaccine has been reported to be a very potent adjuvant [10] and our modeling approach thus confirms the increased proliferation of T-cells for the Shingrix vaccine.

We also assessed whether a correlation existed between the B-cell and T-cell 
counts, but we did not find a significant association between the two immune response types. This confirms previous findings concerning the glycoprotein$\mathrm{E}$ adjuvant, part of the $\mathrm{AS} 1_{B}$ Adjuvant System family, in which it has been shown that this family has been reported to show the lowest correlations between B-cells and T-cells of all families [11].

During our modeling analyses we encountered several limitations. First, we noted that given the limited sample size only models with moderate complexity could be analysed. Second, the sparseness of time points for the B-cell responses posed a significant limitation on the complexity of the B-cell models. Future work should focus on estimating an optimal sampling schedule for subsequent modeling.

In this study, we wanted to focus on the advantages and possibilities of ODE modeling combined with a mixed effect approach in the analysis of vaccine trial immunogenicity data, rather than group-wise or time point-wise comparisons using standard comparative statistics between different vaccination schedules. The techniques underlying this work can now be applied on novel datasets to answer fundamental questions on the understanding of immune responses.

We conclude that nonlinear mixed modeling by means of ODE shows that Shingrix vaccination causes a significantly higher proliferation of T-cells compared to Varilrix vaccination in VZV-immune adults. 


\section{Appendix A. Detailed ODE models and solutions}

\section{Appendix A.1. Antibody secreting cell models}

The dynamics of the memory B-cell population is described using the following ODE:

$$
\frac{d B}{d t}=f_{1}(B) I_{t \leq h}-f_{2}(B)
$$

where $B_{0}=B(0)$ denotes the initial number of memory B-cells at time $=$ 0 (months) and $f_{1}$ and $f_{2}$ are smooth functions of the number of B-cells at time $t$ (months) and describe the change in the number of B-cells due to activation and decay of B-celms. We assume that the activation of B-cells happens during a certain time period $[0, h]$ and that after this time period, no new B-cells are activated. The process of activation of B-cells is thereby described by the function $f_{1}$. The decay of B-cells happens at all times and is described by $f_{2}$. In all models, the decay rate is assumed to remain constant over time and the decay of B-cells is thereby proportional to the number of B-cells.

In model B1, the activation of B-cells is assumed to be constant in time, with $a B$ the rate of activation. This means functions $f_{1}$ and $f_{2}$ can be expressed as

$$
\left\{\begin{array}{l}
f_{1}(B)=a B \\
f_{2}(B)=-u B \cdot B
\end{array}\right.
$$

${ }_{447}$ The solution of this differential equation leads to the following equation

$$
\begin{cases}B(t)=\frac{a B}{u B}+e^{-u B \cdot t}\left(B_{0}-\frac{a B}{u B}\right) & t \leq h \\ B(t)=\left(\frac{a B}{u B}+e^{-u B \cdot h}\left(B_{0}-\frac{a B}{u B}\right)\right) e^{-u B(t-h)} & t>h\end{cases}
$$


454

In model B2, the proliferation of B-cells is assumed to be proportional to the number of $\mathrm{B}$-cells, so the activation is now equal to $p B \times B$ with $p B$ the activation rate. This yields equations

$$
\left\{\begin{array}{l}
f_{1}(B)=p B \cdot B \\
f_{2}(B)=-u B \cdot B
\end{array}\right.
$$

with solution

$$
\begin{cases}B(t)=B_{0} e^{(p B-u B) t} & t \leq h \\ B(t)=B_{0} e^{p B \cdot h-u B \cdot t} & t>h\end{cases}
$$

Previous two models can be combined in the following model, where the activation has both a constant and a proportional component:

$$
\left\{\begin{array}{l}
f_{1}(B)=a B+p B \cdot B \\
f_{2}(B)=-u B \cdot B
\end{array}\right.
$$

with solution

$$
\left\{\begin{array}{rl}
B(t)=-\frac{a B}{p a S C-u B}+\left(B_{0}+\frac{a B}{p B-u B}\right) e^{(p B-u B) t} & t \leq h \\
B(t)=\left(-\frac{a B}{p B-u B}+\left(B_{0}+\frac{a B}{p B-u B}\right) e^{(p B-u B) h}\right) \cdot & \\
e^{-u B(t-h)} & t>h
\end{array}\right.
$$

Since this model did not yield converging results, it was thereby omitted from the paper.

In a next step we distinguish the short-living B-cells $S B(t)$ from the B-cells with long lifespan $L B(t)$. The total number of B-cells is then equal to the sum of these two. We assume that, at baseline, only long-living and thus no 
460

short-living B-cells are present. This model can be described as

$$
\left\{\begin{array}{l}
\frac{d S B}{d t}=f_{1}(S B) I_{t \leq h}+f_{2}(S B) \\
\frac{d L B}{d t}=g_{1}(L B) I_{t \leq h}+g_{2}(L B) \\
B(t)=S B(t)+L B(t)
\end{array}\right.
$$

461

with $L B_{0}=L B(0)$ denoting the initial number of $L B$ and $S B(0)=0$.

First, in model B3 we assume a constant proliferation rate, equal to $a S B$. Furthermore, due to their long lifespan, we assume the decay rate of $L B$ to be equal to zero and that no new long living B-cells are activated. This means the number of $L B$ will remain constant over time. We can summarize this as

$$
\left\{\begin{array}{l}
f_{1}(S B)=a S B S \\
f_{2}(S B)=-u S B \cdot S B \\
g_{1}(L B)=g_{2}(L B)=0
\end{array}\right.
$$

The solution of the ODE of model B3 is given by

$$
\begin{cases}B(t)=-\frac{a S B}{u S B}\left(e^{-u S B \cdot t}-1\right)+L B_{0} & t \leq h \\ B(t)=-\frac{a S B}{u S B}\left(1-e^{u S B \cdot h}\right) e^{-u S B \cdot t}+L B_{0} & t>h\end{cases}
$$

Instead of a constant number of $L B$, we now assume in model B4 that LB will proliferate as well, according to a constant proliferation rate $a L B$ during time period $h$. Their decay will still be neglected due to their long lifespan.

$$
\left\{\begin{array}{l}
f_{1}(S B)=a S B \\
f_{2}(S B)=-u S B \cdot S B \\
g_{1}(L B)=a L B \\
g_{2}(L B)=0
\end{array}\right.
$$


471

with solution

$$
\left\{\begin{array}{cc}
B(t)=-\frac{a S B}{u S B}\left(e^{-u S B \cdot t}-1\right)+L B_{0}+a L B \cdot t & t \leq h \\
B(t)=-\frac{a S B}{u S B}\left(1-e^{u S B \cdot h}\right) e^{-u S B \cdot t}+ & t>h \\
L B_{0}+a L B \cdot h & t>h
\end{array}\right.
$$

Appendix A.2. T-cell models

The design of the T-cell models follows a similar procedure as with the Bcell models. The following differential equation describes the basic dynamics of the stimulus-specific T-cell population:

$$
\frac{d T}{d t}=f_{1}(T) I_{0 \leq t \leq h_{1}}+f_{2}(T) I_{2 \leq t \leq h_{2}}+f_{3}(T)
$$

with $T_{0}=T(0)$ the number of T-cells at time 0 (months). In this equation, $f_{1}(T)$ describes the proliferation of T-cells after the first vaccination event at time 0 , which will occur until a certain time point $h_{1}$ (with $0<h_{1} \leq 2$ ). Afterwards, no T-cells will be activated until the second vaccination event 2 months after the first vaccine, which $f_{2}(T)$ describes as the proliferation of T-cells during the time period $\left[2, h_{2}\right]$, with $h_{2}$ the time point at which the second peak in T-cells is reached. The decay of T-cells will happen during the whole time period, and is represented by the function $f_{3}(T)$. In all models, it will be assumed that the decay rate of T-cells remains constant over time (cfr. B-cell models). Moreover, we assume that the activation of T-cells after each vaccination event happens according to a constant proliferation rate.

Assuming an equal activation rate of $\mathrm{T}$ cells after each vaccination leads to model T1. Functions $f_{i}(i=1,2,3)$ can now be written as

$$
\left\{\begin{array}{l}
f_{1}(T)=f_{2}(T)=a T \\
f_{3}(T)=-u T \cdot T
\end{array}\right.
$$


The solution of the ODE is now given by

$$
\left\{\begin{array}{lll}
T(t)=\frac{a T}{u T}+e^{-u T \cdot t}\left(T_{0}-\frac{a T}{u T}\right) & & t \leq h_{1} \\
T(t)=\left(\frac{a T}{u T}+e^{-u T \cdot h_{1}}\left(T_{0}-\frac{a T}{u T}\right)\right) e^{-u T\left(t-h_{1}\right)} & & h_{1}<t<2 \\
T(t)=\frac{a T}{u T}+\left(T_{0}-\frac{a T}{u T}+\frac{a T}{u T} e^{u T \cdot h 1}-\frac{a T}{u T} e^{u T \cdot 2}\right) e^{-u T \cdot t} & & 2 \leq t \leq h_{2} \\
T(t) & =\left(\frac{a T}{u T}+\left(T_{0}-\frac{a T}{u T}+\frac{a T}{u T} e^{u T \cdot h 1}-\frac{a T}{u T} e^{u T \cdot 2}\right) e^{-u T \cdot h_{2}}\right) \cdot & \\
e^{-u T\left(t-h_{2}\right)} & & t>h_{2}
\end{array}\right.
$$

with solution

$$
\begin{cases}T(t)=\frac{a_{1} T}{u T}+\left(T_{0}+\frac{a_{1} T}{-u T}\right) e^{-u T \cdot t} & t \leq h_{1} \\ T(t)=\left(\frac{a_{1} T}{u T}+\left(T_{0}+\frac{a_{1} T}{-u T}\right) e^{-u T \cdot h_{1}}\right) e^{-u T\left(t-h_{1}\right)} & h_{1}<t<2 \\ T(t)=\frac{a_{2} T}{u T}+\left(\left(\frac{a_{1} T}{u T}+\left(T_{0}-\frac{a_{1} T}{u T}\right) e^{-u T \cdot h_{1}}\right) e^{-u T\left(2-h_{1}\right)}-\frac{a_{2} T}{u T}\right) \cdot & \\ e^{-u T(t-2)} & 2 \leq t \leq h_{2} \\ T(t)=\left(\frac{a_{2} T}{u T}+\left(\left(\frac{a_{1} T}{u T}+\left(T_{0}-\frac{a_{1} T}{u T}\right) e^{-u T \cdot h_{1}}\right) e^{-u T\left(2-h_{1}\right)}-\frac{a_{2} T}{u T}\right) \cdot\right. & \\ \left.e^{-u T(t-2)}\right) e^{-u T\left(t-h_{2}\right)} & t>h_{2}\end{cases}
$$


494 495

Again, in the next step we differentiate between the short living (ST) and long living (LT) T-cells. We express this with equations $f_{i}, g_{i}(i=1: 3)$ :

$$
\left\{\begin{array}{l}
\frac{d S T}{d t}=f_{1}(S T) I_{0<t \leq h_{1}}+f_{2}(S T) I_{2<t \leq h_{2}}+f_{3}(S T) \\
\frac{L S T}{d t}=g_{1}(L T) I_{0<t \leq h_{1}}+g_{2}(L T) I_{2<t \leq h_{2}}+g_{3}(L T) \\
T(t)=S T(t)+L T(t)
\end{array}\right.
$$

with $L T_{0}=L T(0)$ the initial number of long living T-cells and $S T(0)=0$.

As in previous models, we will assume constant proliferation rates $a_{1} S T$ and $a_{2} S T$ of ST, no decay of LT and at first a constant number of LT. Model T3 assumes as well that activation rates of ST after both vaccinations are equal. Functions $f_{i}$ and $g_{i}$ are thereby written as

$$
\left\{\begin{array}{l}
f_{1}(S T)=f_{2}(S T)=a S T \\
f_{3}(S T)=-u S T \cdot S T \\
g_{1}(L T)=g_{2}(L T)=0 \\
g_{3}(L T)=0
\end{array}\right.
$$

501 Its solution is given by

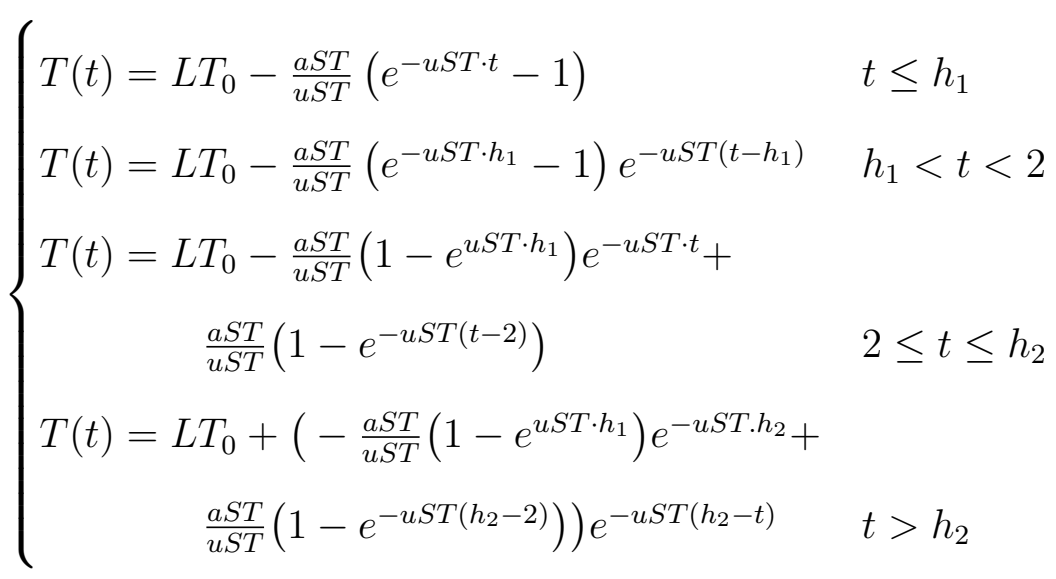


502 Model T4 does not make the assumption of equal ST activation rates, which 503 leads to equations

$$
\left\{\begin{array}{l}
f_{1}(S T)=a_{1} S T \\
f_{2}(S T)=a_{2} S T \\
f_{3}(S T)=-u S T \cdot S T \\
g_{1}(L T)=g_{2}(L T)=0 \\
g_{3}(L T)=0
\end{array}\right.
$$

504

with solution

$$
\begin{cases}T(t)=L T_{0}-\frac{a_{1} S T}{u S T}\left(e^{-u S T \cdot t}-1\right) & t \leq h_{1} \\ T(t)=L T_{0}-\frac{a_{1} S T}{u S T}\left(e^{-u S T \cdot h_{1}}-1\right) e^{-u S T\left(t-h_{1}\right)} & h_{1}<t<2 \\ T(t)=L T_{0}-\frac{a_{1} S T}{u S T}\left(1-e^{u S T \cdot h_{1}}\right) e^{-u S T \cdot t}+ & \\ \quad \frac{a_{2} S T}{u S T}\left(1-e^{-u S T(t-2)}\right) & 2 \leq t \leq h_{2} \\ T(t)=L T_{0}+\left(-\frac{a_{1} S T}{u S T}\left(1-e^{u S T \cdot h_{1}}\right) e^{-u S T \cdot h_{2}}+\right. & \\ \left.\quad \frac{a_{2} S T}{u S T}\left(1-e^{-u S T\left(h_{2}-2\right)}\right)\right) e^{-u S T\left(h_{2}-t\right)} & t>h_{2}\end{cases}
$$

505 Next, we add a constant proliferation rate $a L T$ of long-living T-cells after 506 507 each vaccination A.15a). Model T5 assumes equal activation rates of ST, with following equations:

$$
\left\{\begin{array}{l}
f_{1}(S T)=f_{2}(S T)=a S T \\
f_{3}(S T)=-u S T \cdot S T \\
g_{1}(L T)=g_{2}(L T)=a L T \\
g_{3}(L T)=0
\end{array}\right.
$$


and solution:

$$
\left\{\begin{array}{rlrl}
T(t)=- & \frac{a S T}{u S T}\left(e^{-u S T \cdot t}-1\right)+L T_{0}+a L T . t & & t \leq h_{1} \\
T(t)=- & \frac{a S T}{u S T}\left(e^{-u S T \cdot h_{1}}-1\right) e^{-u S T\left(t-h_{1}\right)}+ & \\
& L T_{0}+a L T \cdot h_{1} & & h_{1}<t<2 \\
T(t)=- & \frac{a S T}{u S T}\left(1-e^{u S T \cdot h_{1}}\right) e^{-u S T \cdot t}+ & \\
& \frac{a S T}{u S T}\left(1-e^{-u S T(t-2)}\right)+ & \\
& & L T_{0}+a L T \cdot\left(h_{1}+t-2\right) & \\
T(t)= & \left(-\frac{a S T}{u S T}\left(1-e^{u S T \cdot h_{1}}\right) e^{-u S T \cdot h_{2}}+\right. & \\
& \left.\frac{a S T}{u S T}\left(1-e^{-u S T\left(h_{2}-2\right)}\right)\right) e^{-u S T\left(h_{2}-t\right)}+ & \\
& & & \\
& &
\end{array}\right.
$$

${ }_{509}$ We conclude the T-cell models with model T6, in which different activation 510 rates of ST after each vaccination are assumed. Equations $f_{i}$ and $g_{i}$ are 511 written as

$$
\left\{\begin{array}{l}
f_{1}(S T)=a_{1} S T \\
f_{2}(S T)=a_{2} S T \\
f_{3}(S T)=-u S T \cdot S T \\
g_{1}(L T)=g_{2}(L T)=a L T \\
g_{3}(L T)=0
\end{array}\right.
$$


512 The solution of the resulting ODE is given by

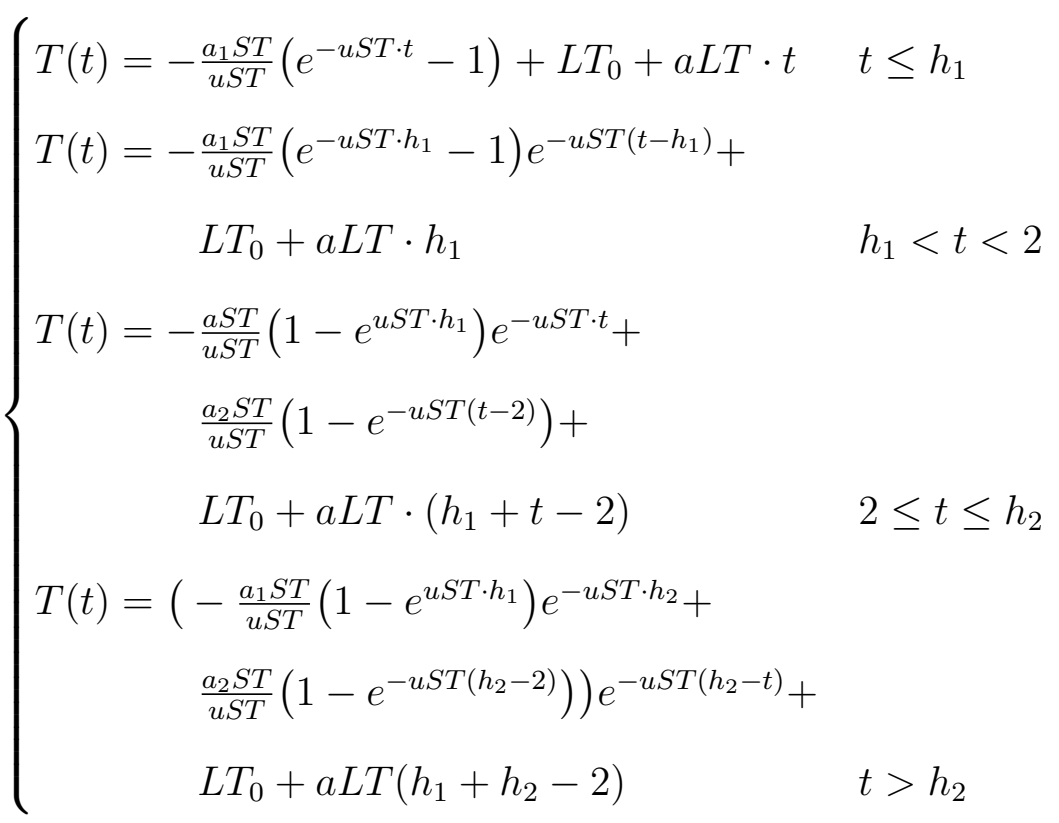




\section{Appendix B. Overview of the different parameters used in the B- cell and T-cell dynamic models}

Table B.7 summarizes the parameters used in the functions of the different B-cell models. Table B.8 summarizes the parameters used in the functions of the different T-cell models.

\begin{tabular}{|l|l|}
\hline Parameter & Description \\
\hline \hline$B_{0}$ & initial number of B-cells \\
\hline$a B$ & constant proliferation rate of B-cells \\
\hline$a S B$ & constant proliferation rate of SB (in case of a distinction) \\
\hline$a L B$ & constant proliferation rate of LB (in case of a distinction) \\
\hline$p B$ & proportional proliferation rate of B-cells \\
\hline$u B$ & decay of (short living, in case of a distinction) B-cells \\
\hline$h$ & time point after which no new B-cells are activated \\
\hline
\end{tabular}

Table B.7: An overview of the different parameters in the B-cell dynamic models 


\begin{tabular}{|l|l|}
\hline Parameter & Description \\
\hline \hline$T_{0}$ & initial number of T-cells \\
\hline$a_{T}$ & \begin{tabular}{l} 
constant proliferation rate of T-cells after the $i$ th vaccination \\
\hline$a_{S} T$
\end{tabular} \\
\hline$a L T$ & $\begin{array}{l}\text { constant proliferation rate of short living T-cells (in case of a } \\
\text { distinction) after } i \text { th vaccination }\end{array}$ \\
\hline$u T$ & distinction) \\
\hline$h_{i}$ & $\begin{array}{l}\text { decay of (short living, in case of a distinction) T-cells } \\
\text { vaccination }\end{array}$ \\
\hline
\end{tabular}

Table B.8: An overview of the different parameters in the T-cell dynamic models 


\section{Appendix C. Algorithm parameter values used in Monolix}

The following list shows a summary of the values used in the SAEMMCMC algorithm and loglikelihood estimation in Monolix:

\begin{tabular}{|c|c|c|}
\hline Population parameters & $\begin{array}{l}\text { SAEM } \\
\text { MCMC } \\
\text { Simulated annealing }\end{array}$ & $\begin{array}{l}K_{0}=100 \\
K_{1}=10^{6} \\
K_{2}=10^{5} \\
a_{1}=0 \\
a_{2}=1 \\
m_{1}=2 \\
m_{2}=0 \\
m_{3}=2 \\
m_{4}=2 \\
\rho=0.3 \\
\tau_{1}=0.95 \\
\tau_{2}=0.95\end{array}$ \\
\hline Individual parameters & $\begin{array}{l}\text { MCMC } \\
\text { Stopping rule }\end{array}$ & $\begin{array}{l}m_{1}=2 \\
m_{2}=0 \\
m_{3}=2 \\
m_{4}=2 \\
\rho=0.3 \\
L_{m c m c}=1000 \\
r_{m c m c}=0.01\end{array}$ \\
\hline Loglikelihood & Importance sampling & Monte-Carlo size $=10^{7}$ \\
\hline
\end{tabular}




\section{Appendix D. B-cell and T-cell dynamics associations and dataset correlations}

\section{Appendix D.1. B-cell and T-cell dynamics associations}

Since the process of B-cell activation is dependent on certain cytokineexpressing T-cells (such as CD40L), the hypothesis is investigated whether the increase in B-cells is proportional to the increase in T-cells. In order to examine this, we define $T 01:=T(1)-T(0)$ and $B 01:=B(1)-B(0)$ and use the minerva package in $\mathrm{R}$ to calculate the maximal information coefficient (MIC). This is a way to detect linear and non-linear relations between variables, and can thereby be used to indicate whether a linear relation is feasible by comparing it with the $\mathrm{R}$-squared value. In addition we compute the Spearman correlation.

We started by examining the Varilrix-specific B-cell and T-cell datasets. The datasets were restricted to individuals without missing values of B-cell or T-cell data at time points 0 and 1 month, 96 individuals in total. First, the hypothesis was made that an increase in T-cells was proportional to an increase in B-cells. We express the expected proportionality factor by $m$ :

$$
m=E\left(\frac{B(1)-B(0)}{T(1)-T(0)}\right) .
$$

Fig 5 shows a scatterplot of $T_{01}:=T(1)-T(0)$ plotted against $B_{01}:=$ $B(1)-B(0)$. At first sight, a linear relation between $T_{01}$ and $B_{01}$ might not seem a reasonable assumption.

To further examine this, we calculated the Spearman correlation and the maximal information coefficient (MIC) as a way to assess and measure (non)linear relationships between datasets. 


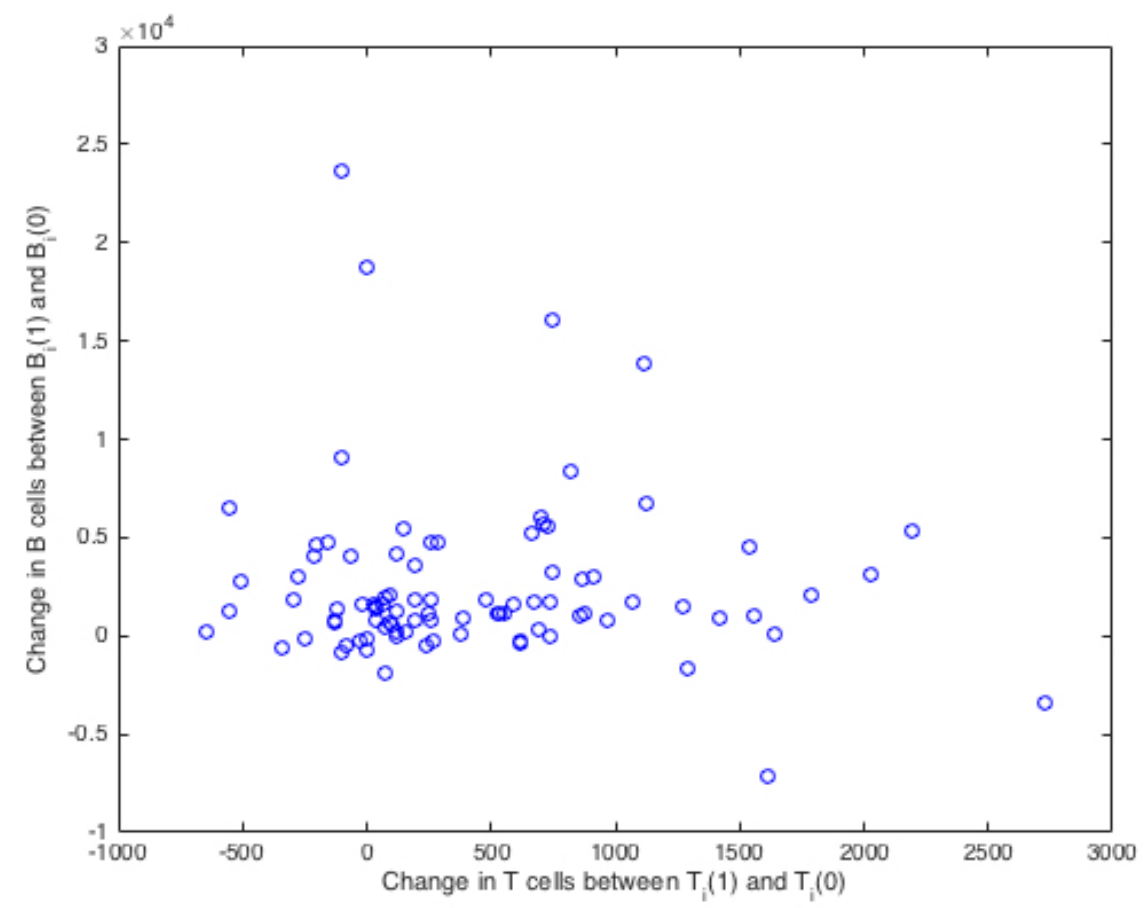

Figure D.5: Scatterplot of the increase in Varilrix-specific T-cells $\left(T_{01}\right)$, plotted against the increase in Varilrix-specific B-cells $\left(B_{01}\right)$. 
The Spearman correlation between Varilrix-specific $T_{01}$ and $B_{01}$ was $0.0274(p=0.7914)$, which rejected the hypothesis that increases in Varilrixspecific T-cells were associated to increases in Varilrix-specific B-cells. A non-significant MIC of 0.2161 (5\% significance level) confirmed this result. We also assessed MIC and Spearman correlations on the same datasets per subgroup, reaching the same conclusion (Table D.9).

\begin{tabular}{|l||l|l||l|l|}
\hline group & MIC & p-value & Spearman & p-value \\
\hline \hline 3 & 0.2854 & $>0.05$ & 0.2096 & 0.2663 \\
\hline 4 & 0.1673 & $>0.05$ & -0.2192 & 0.2271 \\
\hline 5 & 0.2611 & $>0.05$ & 0.1009 & 0.6380 \\
\hline
\end{tabular}

Table D.9: Correlations between B-cells and T-cells (Varilrix stimulus). MIC coefficients, Spearman correlation and corresponding p-values between increase in Varilrixspecific B-cells $\left(B_{01}\right)$ and increase in Varilrix-specific T-cells $\left(T_{01}\right)$, calculated for groups 3,4 and 5. As the sample sizes of groups 1 and 2 were too small $\left(n_{1}=4\right.$ and $\left.n_{2}=6\right)$, those groups were omitted from the analysis.

Potential associations between increases in gE-specific T-cells and B-cells were investigated. The scatterplot of $T_{01}$ plotted against $B_{01}$ is shown in Figure D.6. As before, we also studied the relations between $T_{01}$ and $B_{01}$ per subgroup (see Table D.10). The Spearman p-values showed that only in group 3 the Spearman correlation could be considered significant, together with MIC, implying a nonlinear relationship. The Spearman correlation of $0.3833\left(p=1.0647 e^{-04}\right)$ suggested there was indeed an association. The MIC score was calculated as 0.4107 , which was significant $(p<0.001)$. In case of a linear relation, the R-square is expected to be close to this MIC score. As the R-square was equal to 0.05593 , we could exclude a linear relationship. 


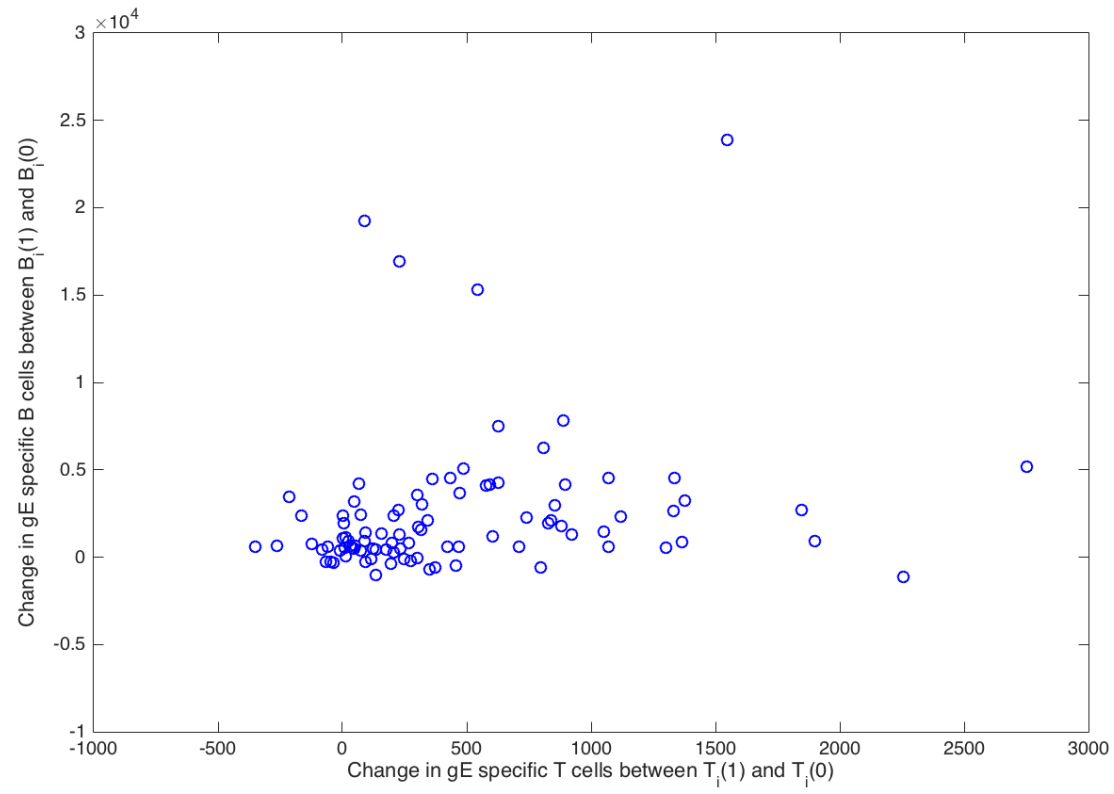

Figure D.6: Scatterplot of the increase in gE-specific T-cells $\left(T_{01}\right)$, plotted against the increase in $\mathrm{gE}$-specific B-cells $\left(B_{01}\right)$. 


\begin{tabular}{|l||l|l|l||l|l|}
\hline group & MIC & p-value & MIC- $R^{2}$ & Spearman & p-value \\
\hline \hline 3 & 0.4734 & 0.01007 & 0.4665 & 0.4725 & 0.0084 \\
\hline 4 & 0.3052 & $>0.05$ & & -0.2300 & 0.2053 \\
\hline 5 & 0.3149 & $>0.05$ & & 0.3138 & 0.1266 \\
\hline
\end{tabular}

Table D.10: Correlations between B-cells and T-cells (gE stimulus). MIC coefficients, Spearman correlation and corresponding p-values between increase in gE-specific B-cells $\left(B_{01}\right)$ and increase in gE-specific T-cells $\left(T_{01}\right)$, calculated for groups 3,4 and 5 . As the sample sizes of groups 1 and 2 were too small $\left(n_{1}=4\right.$ and $\left.n_{2}=6\right)$, those groups were omitted from the analysis.

\section{Appendix D.2. Correlations between B-cell and T-cell datasets}

Instead of solely examining the increase in B-cells and T-cells, we also looked at the correlation between the specific values of B- and T-cells at time points 0 and 1; Spearman correlations for all individuals were calculated between the data points $\mathrm{B}(0), \mathrm{B}(1), \mathrm{T}(0)$ and $\mathrm{T}(1)$.

Correlations between the initial number of $\mathrm{B}$-cells $\mathrm{B}(0)$, the initial number of T-cells $\mathrm{T}(0)$, the number of B-cells at month $1 \mathrm{~B}(1)$ and the number of T-cells at month $1 \mathrm{~T}(1)$ have also been investigated.

We started with the Varilrix-specific B-cell and T-cell data. Again, we only included individuals for whom we had data points $\mathrm{B}(0), \mathrm{B}(1), \mathrm{T}(0)$ and $\mathrm{T}(1)$. Spearman correlations for all individuals were calculated between these data points, and the results are shown in Fig D.7. This figure also shows the Spearman correlations when we separated the individuals by group. When looking at the correlations of all individuals, we noticed correlations between $\mathrm{B}(0)$ and $\mathrm{B}(1)$, and $\mathrm{T}(0)$ and $\mathrm{T}(1)$, but no significant correlations between B- and T-cells. Examining the correlations by group, it seemed those differ 
greatly depending on the group. However, it has to be noted that group 1 and 2 contain a very small number of individuals (4 and 6 individuals respectively). For groups 3 to 5 , the results were similar to the results of the correlation between all individuals, and we therefore found no convincing evidence for an association between Varilrix specific B-cells and T-cells.

The correlation between data points at time 0 and 1 of gE-specific B-cells and T-cells was examined next. Fig D.8 shows the Spearman correlations, first for all individuals and then split by group. We observed that some correlations seemed to be higher compared to the Varilrix-specific data, however, when examining the Spearman matrices by group, the values between the different groups seemed to vary widely. For this reason, we did not find decisive evidence to include the number of $\mathrm{gE}$-specific T-cells into the B-cell models or vice versa.

Appendix D.3. Influence of the initial number of B-cells to the remaining B-cell data

Apart from associations between the B-cell and T-cell datasets, it was investigated as well whether the initial number of B-cells $(B(0))$ had an influence on the short term number of B-cells $(B(1))$, the long term number $(B(12))$ and the decay of B-cells $(B(12)-B(1))$. This was achieved by plotting for each individual $B(0)$ first against $B(1)$, then against $B(12)$ and lastly against $B(12)-B(1)$. If $B(0)$ had influence on either one of these values, a linear relation should be clear from the plot. We started with the Varilrix-specific B-cell data. As can be perceived from Fig D.9, no clear linear relation was found. The same values were plotted afterwards, but now on a logarithmic scale. An evident linear relation could not be detected either. 

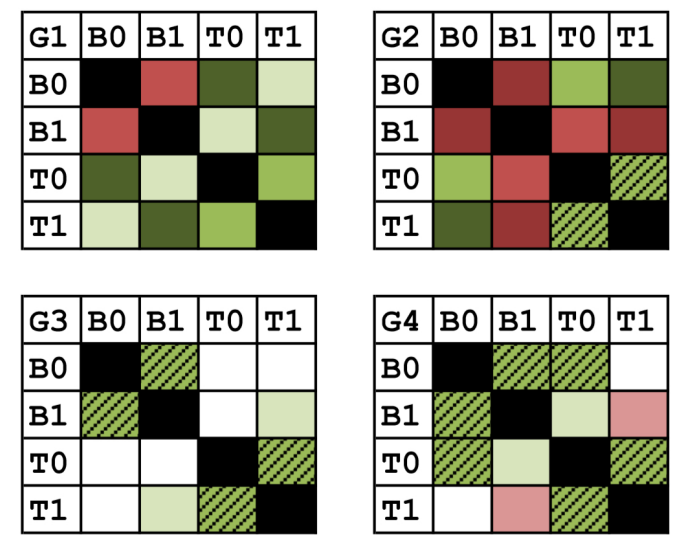

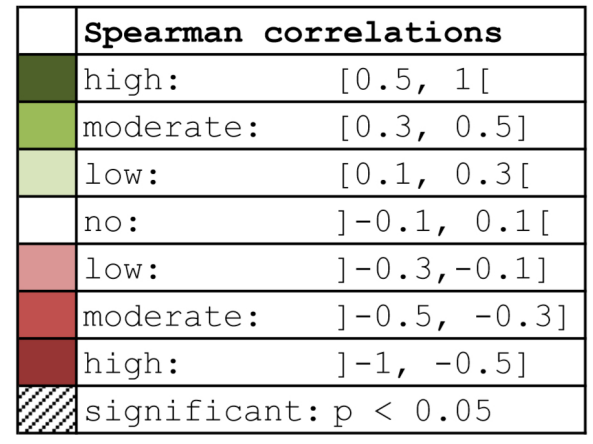
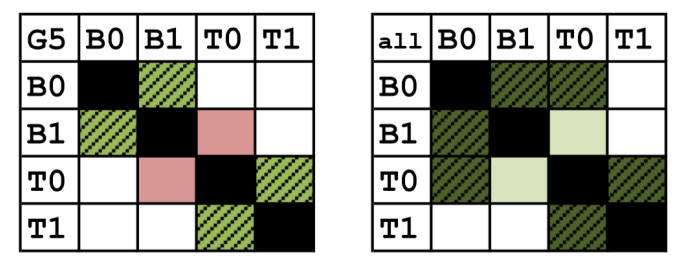

Figure D.7: Spearman correlation between Varilrix-specific data points $B(0)$, $\mathbf{B}(1), \mathbf{T}(0)$ and $\mathbf{T}(1)$, shown for all individuals and per group. Significant correlations are indicated. 

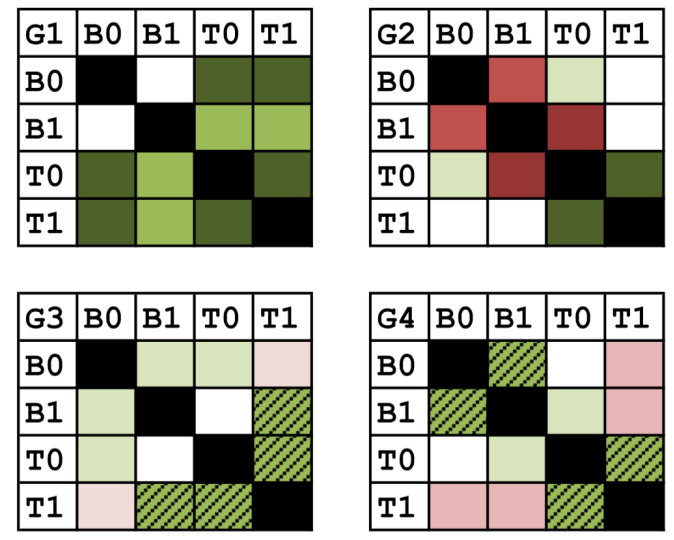

\begin{tabular}{|l|lc|}
\hline & \multicolumn{4}{|l|}{ Spearman correlations } \\
\hline & high: & {$[0.5,1[$} \\
\hline & moderate: & {$[0.3,0.5]$} \\
\hline & low: & {$[0.1,0.3[$} \\
\hline no: & ]$-0.1,0.1[$ \\
\hline low: & ]$-0.3,-0.1]$ \\
\hline & moderate: & ] $-0.5,-0.3]$ \\
\hline & high: & ] $-1,-0.5]$ \\
\hline
\end{tabular}
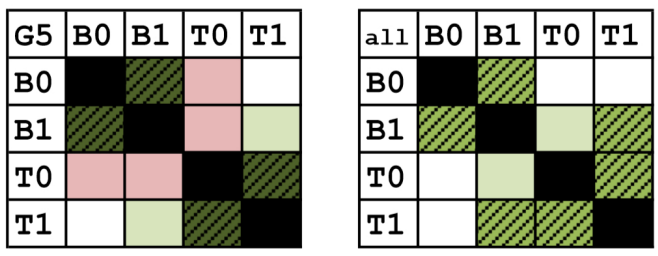

Figure D.8: Spearman correlation between gE-specific data points $\mathrm{B}(0), \mathbf{B}(1)$, $\mathbf{T}(0)$ and $\mathbf{T}(1)$, shown for all individuals and per group. Significant correlations are indicated. 

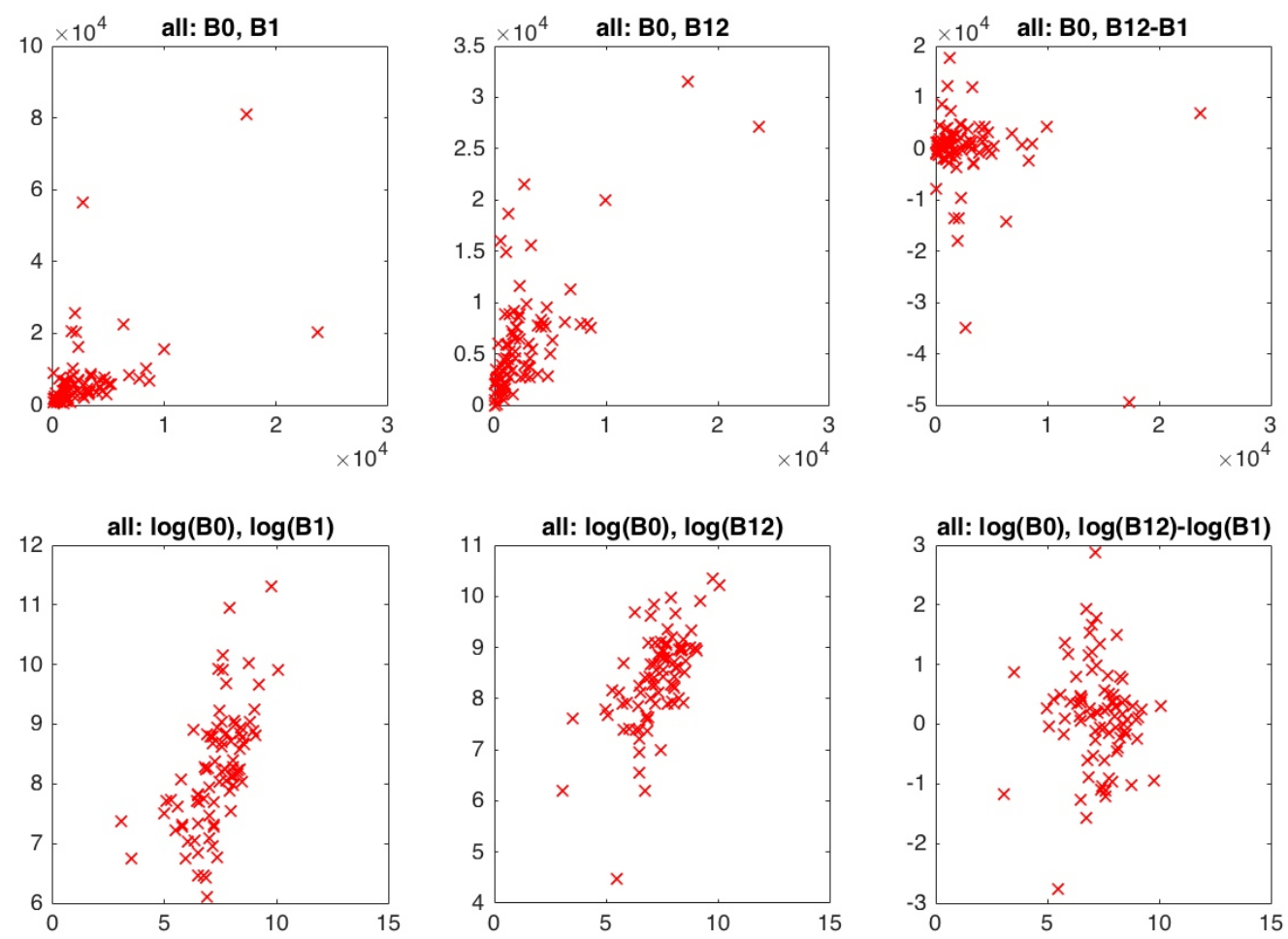

Figure D.9: Associations between Varilrix-specifix B-cell data points. Above: Scatterplots of Varilrix-specific $(\mathrm{B}(0), \mathrm{B}(1)),(\mathrm{B}(0), \mathrm{B}(12)),(\mathrm{B}(0), \mathrm{B}(12)-\mathrm{B}(1))$. Under: Scatterplots of $(\mathrm{B}(0), \mathrm{B}(1)),(\mathrm{B}(0), \mathrm{B}(12)),(\mathrm{B}(0), \mathrm{B}(12)-\mathrm{B}(1))$, on logarithmic scale

Fig D.10 examines the influence of the initial gE-specific B-cell value on the number of short term B-cells, long term and decay of B-cells. There seemed to be even less evidence to assume a linear relation between B-cell values compared to the Varilrix-specific B-cells, on logarithmic scale neither. We can conclude the initial value of B-cells has no definite influence on following B-cell values. 

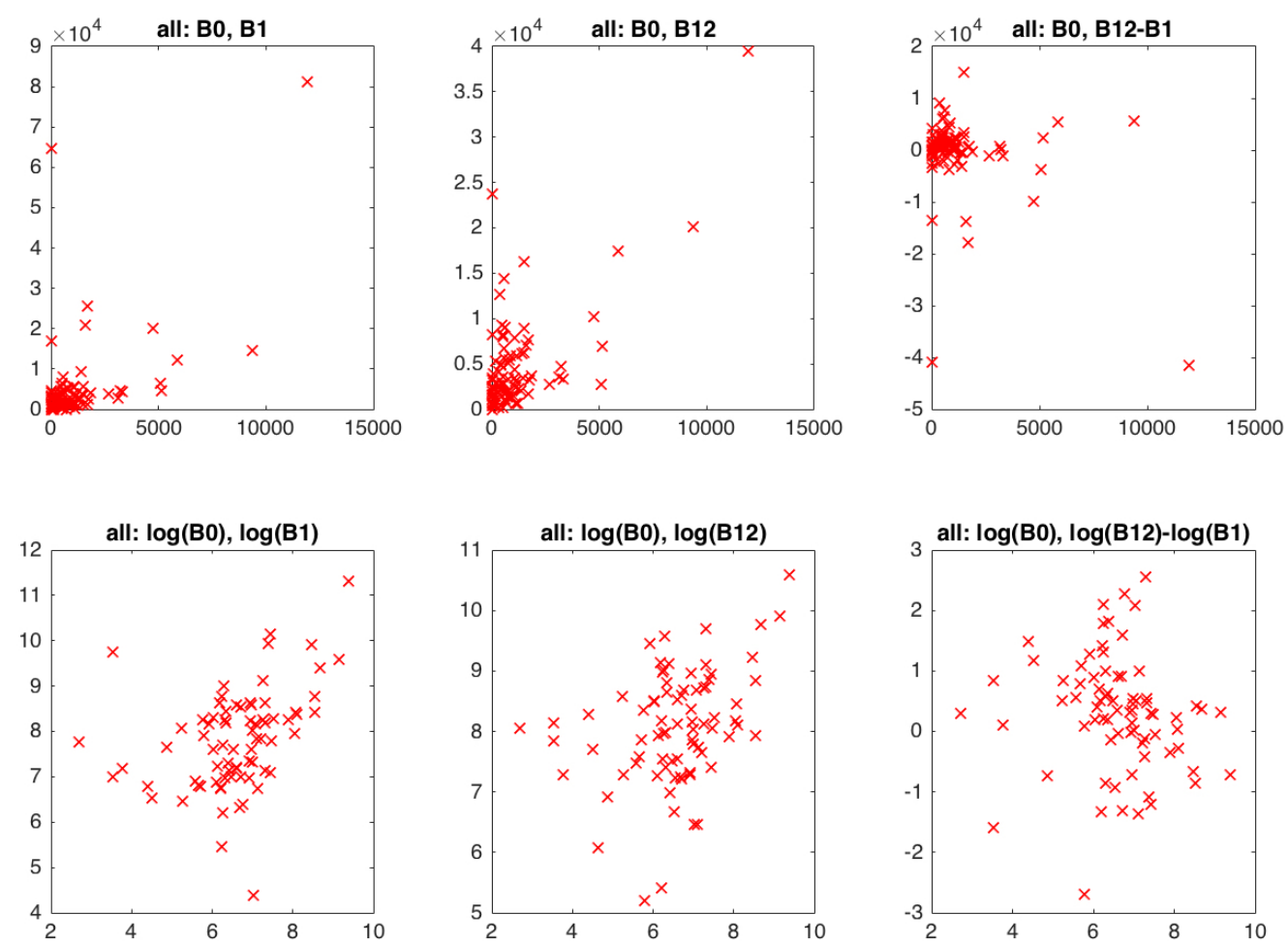

Figure D.10: Associations between gE-specifix B-cell data points. Above: Scatterplots of gE-specific $(\mathrm{B}(0), \mathrm{B}(1))$, ( $\mathrm{B}(0), \mathrm{B}(12))$, ( $\mathrm{B}(0), \mathrm{B}(12)-\mathrm{B}(1))$ Under: Scatterplots of $(\mathrm{B}(0), \mathrm{B}(1)),(\mathrm{B}(0), \mathrm{B}(12)),(\mathrm{B}(0), \mathrm{B}(12)-\mathrm{B}(1))$, on logarithmic scale 


\section{Appendix E. Detailed model selection procedures}

\section{Appendix E.1. Model selection of B-cell datasets}

We started by modeling the Varilrix-specific B-cell dataset. The upper part of Table E.11 summarizes the differences between all models that were tested.

\begin{tabular}{|l|l|l|l|}
\hline model & $\begin{array}{l}\text { fixed population } \\
\text { parameter }\end{array}$ & $\begin{array}{l}\text { group-specific } \\
\text { parameter }\end{array}$ & $\begin{array}{l}\text { convergence- } \\
\text { AIC }\end{array}$ \\
\hline \hline B1a & - & aB & 6559 \\
\hline B1b & uB & aB & 6595 \\
\hline B1c & h & aB & no \\
\hline B2 & - & paB & 6603 \\
\hline B3a & - & aSB & no \\
\hline B3b & uB & aSB & 6541 \\
\hline B3c & h & aSB & no \\
\hline B4a(i/ii) & - & aSB / aLB & no / no \\
\hline B4b(i/ii) & uB & aSB / aLB & no / no \\
\hline B4c & actL & aSB & no \\
\hline B4d(i/ii) & h & aSB / aLB & no / no \\
\hline B4e(i/ii) & uB, h & aSB / aLB & no / no \\
\hline B4f & aLB, h & aSB & no \\
\hline B4g & no & aSB & 6524 \\
\hline \hline candidate & bootstrap & bootstrap \\
\hline model & convergence & ID \\
\hline
\end{tabular}




\begin{tabular}{|l|l|l|l|}
\hline B3b & $65 \%$ & ID54 & $73 \%$ \\
\hline
\end{tabular}

Table E.11: ODE Model formulations considered for Varilrix-specific B-cell data and model selection procedure. Upper part: Overview of the different models used to model the Varilrix-specific B-cell data. First column: model identifier. Second column: parameters selected as fixed population parameter. Third column: parameter which is chosen to be group-specific. Fourth column: AIC value of each model, in case of convergence.

Lower part: Overview of the considered candidate models (first column) used in the Varilrix-specific B-cell data model selection procedure. Second column: convergence results of the performed bootstraps. Third column: the results of possible IDs with deviating presence in the converging bootstrap samples. Fourth column: results of a bootstrap performed on the Varilrix-specific B-cell dataset, in case IDs are found.

612

For model B1, we distinguished between the following scenarios: a scenario with random effects for all parameters (model B1a), a scenario in which the decay of B-cells (uB) was assumed having a fixed population parameter (model B1b), and a scenario in which the time period where B-cells were activated (h) was fixed (model B1c). SAEM convergence was obtained for 
models B1a and B1b, with an AIC value of 6559 and 6595, respectively. Model B1c did not converge within $10^{6}+10^{5}$ iterations and was therefore not considered any further.

Next, a proportional proliferation rate was explored in model B2. Model B2 assumed a group specific proliferation parameter paB and all parameters having random effects. For this model, an AIC value of 6603 was obtained. Consequently, we looked at models in which a distinction between SB and LB was made. Model B3a assumed all parameters had random effects. In models B3b and B3c, $\mathrm{BB}$ and $\mathrm{h}$, respectively, were set as fixed population parameter. Model B3b was the only model that showed convergence, with an AIC value of 6541 .

The last model examined was model B4, in which a proliferation rate for LB was added. Many assumptions on the parameters were made; the decay rate of B-cells $(\mathrm{uB})$, proliferation rate of $\mathrm{LB}(\mathrm{aLB})$ and activation period (h) were set as fixed parameters in models B4b, B4c and B4d, respectively. Combinations of these fixed parameters were considered as well in models B4f, B4g and B4h. Apart from this, we also looked at different group specific parameters, not only the proliferation rate of SB (aSB) was considered, but the proliferation rate of long living B-cells (aLB) as well. Model B4g was the only model that accounted for aSB and aLB and still converged. In this model, both aLB and $\mathrm{uB}$ were set as fixed population parameters, and aSB was considered to be a group specific parameter. This model had the lowest AIC value of 6524 among all aforementioned models, and was selected as first candidate model.

The bootstrap that subsequently was performed did not converge for model 
B4g, and it was therefore in the end rejected.

Model B3b was selected as next-candidate model. The converging of its bootstrap was successful, with $65 \%$ of bootstrap samples showing proper SAEM convergence. An analysis was done to explore whether the presence (or absence) of certain individuals was responsible for the convergence of the datasets. When examining percentages of presence, ID54 showed deviant behavior: the profile was absent from a significant number of the nonconverging datasets. It was therefore removed from the B-cell dataset, after which a new bootstrap with the candidate model was performed. Bootstrap convergence remained well (73\% of bootstrap samples showed SAEM convergence) and model B3b was therefore selected as final Varilrix-specific B-cell model. Model B3b differentiates between SB and LB, LB are assumed to remain constant through time. In time period $(0, h)$, a constant number of $\mathrm{SB}$ is activated and this proliferation rate is considered a group-specific parameter. The decay rate is assumed to be equal for each individual. This model selection procedure is summarized in the lower part of Table E.11.

In the upper part of Table E.12, models and convergence results are shown. Model B3a proved to be the model with lowest AIC value that still provided sufficient bootstrap convergence ( $68 \%$ of all datasets had good SAEM convergence, see lower part of Table E.12). Next, it was investigated whether the convergence for the bootstrap samples was influenced by the presence or absence of certain individual profiles. Indeed, it was discovered that $73 \%$ of the bootstrap samples resulting in non-converge, included ID48 in their dataset. For this reason, a new bootstrap was performed with model B3a, but now on the gE-specific B-cell dataset, excluding ID48. Bootstrap 
convergence was no longer reached and model B3a was discarded. Model B1a, which also showed sufficient bootstrap convergence (68\% of bootstrap samples converged) was considered thereafter. As with previous model, we investigated the relation between the bootstrap convergence and presence or absence of individual profiles. No deviant occurrence was found and model B1a was thereby selected as final gE-specific B-cell model.

\begin{tabular}{|l|l|l|l|}
\hline model & $\begin{array}{l}\text { fixed population } \\
\text { parameter }\end{array}$ & $\begin{array}{l}\text { group-specific } \\
\text { parameter }\end{array}$ & $\begin{array}{l}\text { convergence- } \\
\text { AIC }\end{array}$ \\
\hline \hline B1a & - & aB & 6233 \\
\hline B1b & uB & aB & 6267 \\
\hline B1c & h & aB & 6306 \\
\hline B2 & - & paB & 6304 \\
\hline B3a & - & aSB & 6227 \\
\hline B3b & uB & aSB & 6231 \\
\hline B3c & h & aSB & no \\
\hline B4a(i/ii $)$ & - & aSB / aLB & $6222 / 6207$ \\
\hline B4b(i/ii) & uB & aSB / aLB & no / no \\
\hline B4c & actL & aSB & no \\
\hline B4d(i/ii $)$ & h & aSB / aLB & no / 6257 \\
\hline B4e(i/ii $)$ & uB, h & aSB / aLB & $6267 / 6246$ \\
\hline B4f & aLB, hB & aSB & no \\
\hline B4g & & 6222 \\
\hline
\end{tabular}




\begin{tabular}{|l|l|l|l|}
\hline $\begin{array}{l}\text { candidate } \\
\text { model }\end{array}$ & $\begin{array}{l}\text { bootstrap } \\
\text { convergence }\end{array}$ & $\begin{array}{l}\text { deviating } \\
\text { IDs }\end{array}$ & $\begin{array}{l}\text { bootstrap } \\
\text { w/o ID }\end{array}$ \\
\hline \hline $\mathrm{B} 4 \mathrm{a}(\mathrm{ii})$ & no & & \\
\hline $\mathrm{B} 4 \mathrm{a}(\mathrm{i})$ & no & & \\
\hline $\mathrm{B} 4 \mathrm{~g}$ & no & & \\
\hline $\mathrm{B} 3 \mathrm{a}$ & $68 \%$ & ID48 & no \\
\hline $\mathrm{B} 3 \mathrm{~b}$ & no & & \\
\hline $\mathrm{B} 1 \mathrm{a}$ & $68 \%$ & no & \\
\hline
\end{tabular}

Table E.12: ODE Model formulations considered for gE-specific B-cell data and model selection procedure. Upper part: Overview of the different models used to model the gE-specific B-cell data. First column: model identifier. Second column: parameters selected as fixed population parameter. Third column: parameter which is chosen to be group-specific. Fourth column: AIC value of each model, in case of convergence. Lower part: Overview of the considered candidate models (first column) used in the gE-specific B-cell data model selection procedure. Second column: convergence results of the performed bootstraps. Third column: the results of possible IDs with deviating presence in the converging bootstrap samples. Fourth column: results of a bootstrap performed on the gE-specific B-cell dataset, in case IDs are found. 
Appendix E.2. Model selection of T-cell datasets

Just like with the B-cell models, the most simplistic T-cell model T1 was considered first, in which $a_{1} T=a_{2} T=a T$. Model T1a assumed all parameters had random effects, and the activation of T-cells $(a T)$ had a group-specific effect. An AIC value of 11,664 was obtained.

When assuming $a_{1} T \neq a_{2} T$, we arrived at model T2. First, the assumption was made that all parameters had random effects, and both $a_{1} T$ and $a_{2} T$ had a group-specific effect in model T2a. This model converged, with an AIC value of 11,658 . When assuming only $a_{2} T$ had a group-specific effect (model T2b), a slightly lower AIC value was obtained at 11,655.

Next, a distinction between short and long living T-cells (ST and LT, respectively) was considered. In model T3a, all parameters had random effects and $a S T\left(=a_{1} S T=a_{2} S T\right)$ had a group-related effect resulting in an AIC value of 11,637. Model T3b, in which $u T$ was a fixed parameter, did not reach convergence.

Model T4 assumed different activation rates of T-cells after each vaccination. When all parameters had random effects, and both $a_{1} T$ and $a_{2} T$ were group specific, SAEM convergence was not reached (model T4a). When only a group specific effect on $a_{2} T$ was assumed (model T4b), convergence was achieved resulting in an AIC value of 11,615. Subsequently, $u T$ was set as fixed parameter in models $\mathrm{T} 4 \mathrm{c}$ and $\mathrm{T} 4 \mathrm{~d}$, again with group specific effects on both activation rates (T4c) and on $a_{2} T$ only (T4d), respectively. Both models showed SAEM convergence with an AIC value of 11,646 and a lower AIC value of 11,626, respectively.

When assuming LT activation according to a constant proliferation rate 
(equal after each vaccination in order to limit the number of parameters to be estimated), models $\mathrm{T} 5$ and $\mathrm{T} 6$ were reached. In models $\mathrm{T} 5$, the activation rates of ST were presumed equal after each vaccination. Together with the assumption that all parameters were random, and $a S T$ was a group specific parameter, this leaded to model T5a, where an AIC value of 11,631 was found. We note that setting $a L T$ as a group specific parameter was tested as well, but none of these models (including the following) showed convergence and thus were omitted from Table E.13.

\begin{tabular}{|l|l|l|l|}
\hline model & $\begin{array}{l}\text { fixed population } \\
\text { parameter }\end{array}$ & $\begin{array}{l}\text { group-specific } \\
\text { parameter }\end{array}$ & $\begin{array}{l}\text { convergence- } \\
\text { AIC }\end{array}$ \\
\hline \hline T1a & - & $a T$ & 11664 \\
\hline T2a & - & $a_{1} T, a_{2} T$ & 11658 \\
\hline T2b & - & $a_{2} T$ & 11655 \\
\hline T3a & - & $a S T$ & 11637 \\
\hline T3b & $u T$ & $a S T$ & no \\
\hline T4a & - & $a_{1} S T, a_{2} S T$ & no \\
\hline T4b & - & $a_{2} S T$ & 11615 \\
\hline T4c & $u T$ & $a_{1} S T, a_{2} S T$ & 11646 \\
\hline T4d & $u T$ & $a_{2} S T$ & 11626 \\
\hline T5a & - & $a S T$ & 11631 \\
\hline T5b & $u T$ & $a S T$ & 11637 \\
\hline T5c & $a L T$ & $a S T$ & 11640 \\
\hline T5d & $a L T, u T$ & $a S T$ & 11667 \\
\hline T6a & - & $a_{1} S T, a_{2} S T$ & no \\
\hline
\end{tabular}




\begin{tabular}{|c|c|c|c|}
\hline T6b & - & $a_{2} S T$ & no \\
\hline T6c & $u T$ & $a_{1} S T, a_{2} S T$ & no \\
\hline T6d & $u T$ & $a_{2} S T$ & 11630 \\
\hline T6e & $a L T$ & $a_{1} S T, a_{2} S T$ & no \\
\hline T6f & $a L T$ & $a_{2} S T$ & 11624 \\
\hline T6g & $a L T, u T$ & $a_{2} S T$ & no \\
\hline $\begin{array}{l}\text { candidate } \\
\text { model }\end{array}$ & $\begin{array}{l}\text { bootstrap } \\
\text { convergence }\end{array}$ & $\begin{array}{l}\text { deviating } \\
\text { IDs }\end{array}$ & $\begin{array}{l}\text { bootstrap } \\
\text { w/o ID }\end{array}$ \\
\hline T4b & no & & \\
\hline T6f & no & & \\
\hline $\mathrm{T} 4 \mathrm{~d}$ & no & & \\
\hline T6d & no & & \\
\hline $\mathrm{T} 5 \mathrm{a}$ & no & & \\
\hline $\mathrm{T} 5 \mathrm{~b}$ & no & & \\
\hline T3a & $66 \%$ & no & \\
\hline T3a' & $67 \%$ & no & \\
\hline
\end{tabular}




\section{Table E.13: ODE Model formulations considered}

for Varilrix-specific T-cell data and model selection procedure. Above: Overview of the different models used to model the Varilrix-specific T-cell data. First column: model identifier. Second column: parameters selected as fixed population parameter. Third column: parameter which is chosen to be group-specific. Fourth column: AIC value of each model, in case of convergence. Under: Overview of the considered candidate models (first column) used in the Varilrix-specific T-cell data model selection procedure. Second column: convergence results of the performed bootstraps. Third column: the results of possible IDs with deviating presence in the converging bootstrap samples. Fourth column: results of a bootstrap performed on the Varilrix-specific T-cell dataset, in case IDs are found.

In Models T5b and T5c, respectively, $u T$ and $a L T$ were assumed to be fixed population parameters. They showed SAEM convergence, with AIC values of 11,637 and 11,640, respectively. Setting both $u T$ and $a L T$ fixed in model T5d did not improve the model (AIC: 11,667).

Finally, model T6 was considered, with different activation rates after each vaccination event. Assuming all parameters were random and either both $a_{1} S T$ and $a_{2} S T$ (T6a), or only $a_{2} S T$ (T6b), were group specific, did not lead 
to convergence within $10^{6}+10^{5}$ iterations. For this reason, fixed parameters $u T$ and/or $a L T$ were considered. When $u T$ was fixed, and both activation rates were group specific, convergence was not reached (model T6c). With $a_{2} S T$ being group specific (model T6d), convergence was obtained with an AIC value of 11,630 . In case of setting $a L T$ as a fixed parameter, similar results were found; assuming both activation rates to be group specific did not lead to convergence, but assuming only $a_{2} S T$ was group specific, did, with a slightly lower AIC value equal to 11,624. The last scenario assumed both $a L T$ and $u T$ were fixed population parameters, though no convergence was obtained.

Since model T4b was the model with lowest AIC $(11,615)$, it was selected as first candidate model. However, model T4b was subsequently rejected as a 1000 sample bootstrap failed to converge. Likewise models T6f, T4d, T6d, T5a and T5b did not have proper bootstrap convergence. Next, model T3a was selected as candidate model and showed bootstrap convergence; $66 \%$ of the bootstrap samples reached SAEM convergence. As before, a search for frequently deviant profiles in the converging and non-converging bootstrap datasets was performed, but no such profile was identified.

Taking into account that the assumption that $a_{1} S T=a_{2} S T=a S T$ might not be a realistic assumption in a model that described a real life cellular process, a difference in proliferation rates after each vaccination was inserted, assuming $a_{2} S T$ was proportional to $a_{1} S T: a_{2} S T=k \times a_{1} S T$. Adding this parameter to the pool of parameters to be estimated in the SAEM algorithm, did not yield a converging model. In order to limit the number of parameters 
to be estimated by the SAEM-algorithm, different values of $k$ ware set fixed and for each subsequent model, AIC values were compared. A model with $k=0.15$ showed the lowest AIC value $(11,623)$, which we named model T3a', and a bootstrap with 100 bootstrap samples was performed on this model. From this bootstrap, $67 \%$ of samples resulted in SAEM convergence and a search for disproportionate presence of deviant profile(s) did not identify such profiles. Therefore, model T3a', a model which differentiates between ST and LT, was selected as final Varilrix-specific T-cell model.

Table E.14 summarizes the models used in the selection procedure of gE-specific T-cells. One individual (ID149), with deviating T-cell data, has been left off from the model selection procedure since all models of the dataset including this individual did not converge. Model T1a was the singular model with a converging SAEM algorithm and was thereby selected as candidate model. The bootstrap that was performed afterwards converged as well.

However, it was found that ID89 heavily influenced the converging value of one of the parameters $(h)$. For this reason, ID89 was omitted from the gE T-cell dataset. Without this parameter, the bootstrap convergence of model T1a remained.

In the next step, we included parameter $a_{2} T$ in the model, with $a_{2} T=k \times a_{T}$. $k$ was estimated as 0.66 by comparing AIC-values for models with various values of $k$. The resulting model, T1a', showed bootstrap convergence and the bootstrap samples did not possess deviating presence of certain IDs. For this reason, model T1a' was selected as final gE-specific T-cell model.

\begin{tabular}{|l|l|l|l|}
\hline model & $\begin{array}{l}\text { fixed population } \\
\text { parameter }\end{array}$ & $\begin{array}{l}\text { group-specific } \\
\text { parameter }\end{array}$ & convergence- \\
& AIC \\
\hline
\end{tabular}




\begin{tabular}{|l|l|l|l|}
\hline T1a & - & $a T$ & 11658 \\
\hline T2a & - & $a_{1} T, a_{2} T$ & no \\
\hline T2b & - & $a_{2} T$ & no \\
\hline T3a & - & $a S T$ & no \\
\hline T3b & $u T$ & $a S T$ & no \\
\hline T4a & - & $a_{1} S T, a_{2} S T$ & no \\
\hline T4b & - & $a_{2} S T$ & no \\
\hline T4c & $u T$ & $a_{1} S T, a_{2} S T$ & no \\
\hline T4d & $u T$ & $a_{2} S T$ & no \\
\hline T5a & - & $a_{S T}$ & no \\
\hline T5b & $u T$ & $a S T$ & no \\
\hline T5c & $a L T$ & $a S T$ & no \\
\hline T5d & $a L T, u T$ & $a_{S T}$ & no \\
\hline T6a & - & $a_{1} S T, a_{2} S T$ & no \\
\hline T6b & - & $a_{2} S T$ & no \\
\hline T6c & $u T$ & $a_{1} S T, a_{2} S T$ & no \\
\hline T6d & $u T$ & $a_{2} S T$ & no \\
\hline T6e & $a L T$ & $a_{1} S T, a_{2} S T$ & no \\
\hline T6f & $a L T$ & $a_{2} S T$ & no \\
\hline T6g & $a L T, u T$ & $a_{2} S T$ & no \\
\hline \hline candidate & bootstrap & deviating & bootstrap \\
model & convergence & IDs & w/o ID \\
\hline \hline T1a & yes & no & yes \\
\hline T1a' & yes & \\
\hline
\end{tabular}




\section{Table E.14: ODE Model formulations considered}

for $\mathrm{gE}$-specific $\mathrm{T}$-cell data and model selection procedure. Above: Overview of the different models used to model the gE-specific T-cell data. First column: model identifier. Second column: parameters selected as fixed population parameter. Third column: parameter which is chosen to be group-specific. Fourth column: AIC value of each model, in case of convergence.

Under: Overview of the considered candidate models (first column) used in the gE-specific T-cell data model selection procedure. Second column: convergence results of the performed bootstraps. Third column: the results of possible IDs with deviating presence in the converging bootstrap samples. Fourth column: results of a bootstrap performed on the gE-specific T-cell dataset, in case IDs are found. 


\section{Acknowledgements}

This research was funded by the University of Antwerp [BOF Concerted Research Action, Methusalem funding], NH acknowledges support from the chair in evidence-based vaccinology sponsored by a gift from Pfizer (20092018) and GSK (2016) and from the European Research Council (ERC) under the European Union's Horizon 2020 research and innovation programme (grant agreement 682540 - TransMID). This research was supported by the Antwerp Study Centre for Infectious Diseases (BID). The funders had no role in study design, data collection and analysis, decision to publish, or preparation of the manuscript.

All authors attest they meet the ICMJE criteria for authorship.

\section{References}

[1] Van Herck K, Beutels P, Van Damme P, Beutels M, Van den Dries $\mathrm{J}$, Briantais $\mathrm{P}$, et al. Mathematical models for assessment of long-term persistence of antibodies after vaccination with two inactivated hepatitis A vaccines. Journal of Medical Virology. 2000;60(1):17.

[2] Hens N, Habteab Ghebretinsae A, Hardt K, Van Damme P, Van Herck K. Model based estimates of long-term persistence of inactivated hepatitis A vaccine-induced antibodies in adults. Vaccine. 2014;32(13):15071513. doi:10.1016/j.vaccine.2013.10.088.

[3] Theeten H, Van Herck K, Van Der Meeren O, Crasta P, Van Damme P, Hens N. Long-term antibody persistence after vaccination with a 2dose Havrix (inactivated hepatitis A vaccine): 20 years of observed data, 
and long-term model-based predictions. Vaccine. 2015;33(42):57235727. doi:10.1016/j.vaccine.2015.07.008.

[4] Andraud M, Lejeune O, Musoro JZ, Ogunjimi B, Beutels P, Hens N. Living on three time scales: The dynamics of plasma cell and antibody populations illustrated for hepatitis A virus. PLoS Computational Biology. 2012;8(3):18. doi:10.1371/journal.pcbi.1002418.

[5] Le D, Miller JD, Ganusov VV. Mathematical modeling provides kinetic details of the human immune response to vaccination. Frontiers in Cellular and Infection Microbiology. 2015;4(January):113. doi:10.3389/fcimb.2014.00177.

[6] Leroux-Roels I, Leroux-Roels G, Clement F, Vandepapelire P, Vassilev V, Ledent E, et al. A Phase 1/2 Clinical Trial Evaluating Safety and Immunogenicity of a Varicella Zoster Glycoprotein E Subunit Vaccine Candidate in Young and Older Adults. The Journal of Infectious Diseases. 2012;206(8):12801290. doi:10.1093/infdis/jis497.

[7] Amanna IJ, Slifka MK. Mechanisms that determine plasma cell lifespan and the duration of humoral immunity. Immunological Reviews. 2010;236(1):125138. doi:10.1111/j.1600-065X.2010.00912.x.

[8] Lavielle M, Mentr F. Estimation of population pharmacokinetic parameters of saquinavir in HIV patients with the MONOLIX software. Journal of Pharmacokinetics and Pharmacodynamics. 2007;34(2):229249. doi:10.1007/s10928-006-9043-z. 
806

[9] Reshef D, Reshef Y, Finucane H, Grossman S, Mcvean G, Turnbaugh P, et al. Detecting Novel Associations in Large Data Sets. Science Translational Medicine. 2011;334(6062):15181524. doi:10.1126/science.1205438.

[10] Didierlaurent AM, Laupze B, Di Pasquale A, Hergli N, Collignon C, Garon N. Adjuvant system AS01: helping to overcome the challenges of modern vaccines. Expert Review of Vaccines. 2017;16(1):5563. doi:10.1080/14760584.2016.121363

[11] Leroux-Roels G, Marchant A, Levy J, Van Damme P, Schwarz T, Horsmans $\mathrm{Y}$, et al. Impact of adjuvants on $\mathrm{CD} 4+\mathrm{T}$ cell and $\mathrm{B}$ cell responses to a protein antigen vaccine: Results from a phase II, randomized, multicenter trial. Clinical Immunology. 2016;169(2016):16-27. doi: 10.1016/j.clim.2016.05.007. 


\section{Supplementary material}

Fig S1. Amount of VZV IgG-secreting cells. Measured by Bcell ELISPOT (gE stimulus) per $10^{6}$ IgG-secreting cells, up to 12 months. Data are shown per study group. The last panel acts as an illustration of the vaccination dynamics and shows a hypothetical, smooth function of the expected change in number of memory B-cells over time (in months), based on the observed data points per individual and considering the second vaccination at month 2 .

Fig S2. Amount of gE-specific CD4+ T-cells, producing at least 2 immune markers. Measured by ICS per $10^{6}$ CD $4+$ T-cells, shown per group and up to 12 months. The last panel acts as an illustration of the vaccination dynamics and shows a hypothetical, smooth function of the expected change in number of CD4+ T-cells over time (in months), based on the observed data points per individual.

Dataset 1. Varilrix-specific memory B-cells. Amount of VZVspecific memory B-cells. Measured by B-cell ELISPOT (Varilrix stimulus) per $10^{6}$ total memory cells, up to 12 months.

Dataset 2. gE-specific memory B-cells. Amount of VZV-specific memory B-cells. Measured by B-cell ELISPOT (gE stimulus) per $10^{6}$ total memory cells, up to 12 months.

Dataset 3. Varilrix-specific CD4+ T-cells. Amount of VZV-specific CD4+ T-cells (Varilrix stimulus) producing at least 2 immune markers. Measured by ICS per $10^{6}$ CD $4+$ T-cells up to 12 months.

Dataset 4. gE-specific CD4+ T-cells. Amount of VZV-specific CD4+ T-cells (gE stimulus) producing at least 2 immune markers. Mea- 
${ }_{843}$ sured by ICS per $10^{6}$ CD $4+$ T-cells up to 12 months. 